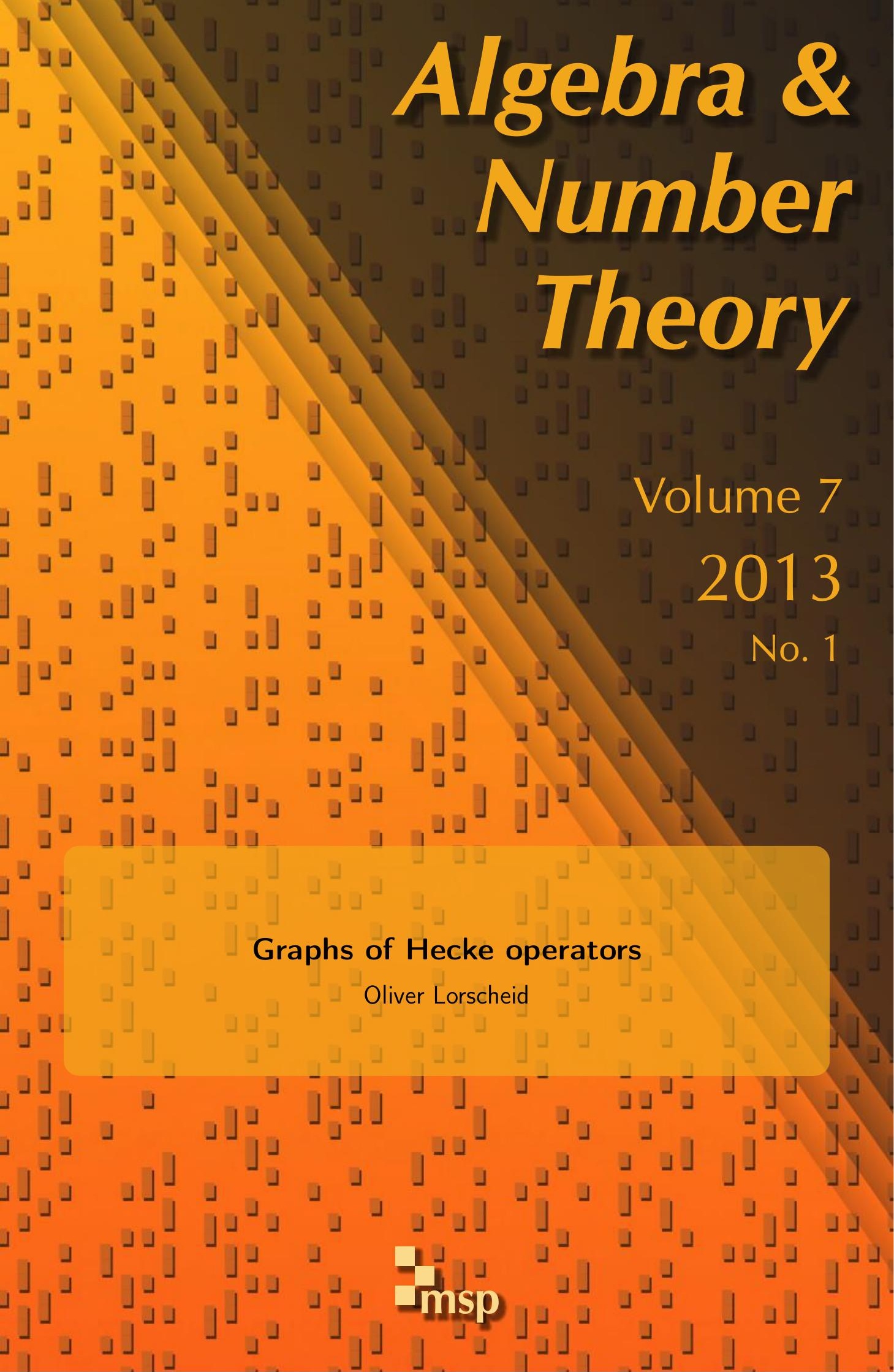




\section{Graphs of Hecke operators}

\section{Oliver Lorscheid}

Let $X$ be a curve over $\mathbb{F}_{q}$ with function field $F$. In this paper, we define a graph for each Hecke operator with fixed ramification. A priori, these graphs can be seen as a convenient language to organize formulas for the action of Hecke operators on automorphic forms. However, they will prove to be a powerful tool for explicit calculations and proofs of finite dimensionality results.

We develop a structure theory for certain graphs $\mathscr{G}_{x}$ of unramified Hecke operators, which is of a similar vein to Serre's theory of quotients of Bruhat-Tits trees. To be precise, $\mathscr{G}_{x}$ is locally a quotient of a Bruhat-Tits tree and has finitely many components. An interpretation of $\varphi_{x}$ in terms of rank 2 bundles on $X$ and methods from reduction theory show that $\mathscr{G}_{x}$ is the union of finitely many cusps, which are infinite subgraphs of a simple nature, and a nucleus, which is a finite subgraph that depends heavily on the arithmetic of $F$.

We describe how one recovers unramified automorphic forms as functions on the graphs $\mathscr{G}_{x}$. In the exemplary cases of the cuspidal and the toroidal condition, we show how a linear condition on functions on $\mathscr{G}_{x}$ leads to a finite dimensionality result. In particular, we reobtain the finite-dimensionality of the space of unramified cusp forms and the space of unramified toroidal automorphic forms.

In an appendix, we calculate a variety of examples of graphs over rational function fields.

Introduction $\quad 20$

1. Definitions 23

2. Unramified Hecke operators 27

3. Connection with Bruhat-Tits trees 28

4. A vertex labeling 30

5. Geometric interpretation of unramified Hecke operators 31

6. Description of vertices 35

7. Reduction theory for rank 2 bundles 38

8. Nucleus and cusps 40

9. Application to automorphic forms 45

10. Finite-dimensionality results 47

MSC2010: primary 11F41; secondary 05C75, 11G20, 14H60, $20 \mathrm{C} 08$.

Keywords: curve over a finite field, vector bundles, automorphic forms, Hecke operator, Bruhat-Tits tree. 
Appendix: Examples for rational function fields

\section{Introduction}

Hecke operators play a central role in the theory of automorphic forms, and for classical modular forms, they are also computationally well understood. The theory of arithmetic quotients of the Bruhat-Tits tree as studied in [Serre 2003] allowed the study of Hecke operators over $p$-adic fields by geometric methods. In this paper, we consider how to compute with Hecke operators for automorphic forms on $\mathrm{PGL}_{2}$ over a global function field. Our theory can be understood as a global counterpart to Serre's viewpoint over $p$-adic fields.

There are a few applications of Serre's theory to automorphic forms over global fields, which, however, mainly concentrate on rational function fields; see [Gekeler 1995; 1997; Gekeler and Nonnengardt 1995]. The key ingredient of this application is the strong approximation property of $\mathrm{SL}_{2}$, as we will explain below. We begin with reminding the reader of the definition of a Bruhat-Tits tree. Though this paper is independent from Serre's book [2003], we review some aspects of it since the global theory (as developed in this paper) and the local approach (as in Serre's book) go hand in hand. In later parts of the paper, we make a few remarks pointing out the connections with and the differences to Serre's theory.

Let $F$ be a global function field and $x$ be a fixed place. We denote by $F_{x}$ the completion of $F$ at $x$, by $O_{x}$ its integers, by $\pi_{x} \in \mathscr{O}_{x}$ a uniformizer and by $q_{x}$ the cardinality of the residue field $\mathcal{O}_{x} /\left(\pi_{x}\right) \simeq \mathbb{F}_{q_{x}}$. The Bruhat-Tits tree $\mathscr{T}_{x}$ of $F_{x}$ is a graph with vertex set $\mathrm{PGL}_{2}\left(F_{x}\right) / \mathrm{PGL}_{2}\left(\mathrm{O}_{x}\right)$. There is an edge between two cosets $[g]$ and $\left[g^{\prime}\right]$ if and only if $\left[g^{\prime}\right]$ contains $g\left({ }^{1} \pi_{x}\right)$ or $g\left(\begin{array}{rl}\pi_{x} & b \\ 1\end{array}\right)$ for some $b \in \mathbb{F}_{q_{x}}$. Note that this condition is symmetric in $g$ and $g^{\prime}$, so $\mathscr{T}_{x}$ is a geometric graph. In fact, $\mathscr{T}_{x}$ is a $\left(q_{x}+1\right)$-regular tree.

Every subgroup of $\mathrm{PGL}_{2}\left(F_{x}\right)$ acts on $\mathscr{T}_{x}$ by multiplication from the left. We shall be interested in the following case. Let $0_{F}^{x} \subset F$ be the Dedekind ring of all elements $a \in F$ with $\|a\|_{y} \leq 1$ for all places $y \neq x$. Put $\Gamma=\operatorname{PGL}_{2}\left(O_{F}^{x}\right)$. Serre [2003] investigates the quotient graph $\Gamma \backslash \mathscr{T}_{x}$. It is the union of a finite connected graph with a finite number of cusps. A cusp is an infinite graph of the form

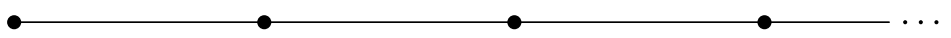

and each cusp corresponds to an element of the class group of $\mathbb{O}_{F}^{x}$.

An unramified automorphic form over $F_{x}$ can be interpreted as a function $f$ on the vertices of $\Gamma \backslash \mathscr{T}_{x}$ such that the space of functions generated by $\left\{T_{x}^{i}(f)\right\}_{i \geq 0}$ is 
finite-dimensional, where the Hecke operator $T_{x}$ is defined by the formula

$$
T_{x}(f)([g])=\sum_{\substack{\text { edges } e \text { with origin }[g] \\ \text { and terminus }\left[g^{\prime}\right]}}\left[\operatorname{Stab}_{\Gamma}([g]): \operatorname{Stab}_{\Gamma}(e)\right] \cdot f\left(\left[g^{\prime}\right]\right)
$$

for each coset $[g] \in \mathrm{PGL}_{2}\left(F_{x}\right) / \mathrm{PGL}_{2}\left(\mathscr{O}_{x}\right)$.

The inclusion of $\mathrm{PGL}_{2}\left(F_{x}\right)$ as $x$-component into $\mathrm{PGL}_{2}(\mathbb{A})$ induces a map

$$
\Gamma \backslash \mathrm{PGL}_{2}\left(F_{x}\right) / \mathrm{PGL}_{2}\left(\mathrm{O}_{x}\right) \rightarrow \mathrm{PGL}_{2}(F) \backslash \mathrm{PGL}_{2}(\mathbb{A}) / \mathrm{PGL}_{2}\left(\mathrm{O}_{\mathrm{A}}\right),
$$

where $\mathscr{O}_{\mathrm{A}}$ is the maximal compact subring of the adeles $\mathbb{A}$ of $F$. In the case that $F$ is a rational function field (as in [Gekeler 1995; 1997; Gekeler and Nonnengardt 1995], or, more generally, a function field with odd class number, and $x$ is a place of odd degree, this map is a bijection as a consequence of the strong approximation property of $\mathrm{SL}_{2}$ (more detail will be given in Section 3). The double coset space on the right hand side is the domain of automorphic forms over $F$, and the bijection is equivariant with respect to the Hecke operator $T_{x}$ and its global equivalent $\Phi_{x}$.

In this sense, it is possible to approximate automorphic forms in this case and use the theory from Serre's book. However, the method of approximation breaks down if the function field has even class number or if the Hecke operator of interest is attached to a place of even degree. For automorphic forms over any function field (with possibly even class number) or for the investigation of Hecke operators at any place of a given function field, respectively, a simultaneous description of all Hecke operators, the method of strong approximation is thus insufficient, and we see the need of a global analogue, which is the starting point of this paper.

The applications of this theory are primarily in explicit computations with automorphic forms. For instance, Lorscheid [2012] uses graphs of Hecke operators to calculate the dimensions of spaces of cusp forms and toroidal automorphic forms. From a more conceptual viewpoint, it might be fruitful to explore the connections between graphs of Hecke operators and Drinfeld modules; in particular, it might contribute to the Langlands program since there is a generalization of graphs of Hecke operator to all reductive groups via adelic Bruhat-Tits buildings, which we forgo explaining here.

We give an overview of the content of this paper. In Section 1, we introduce the graph of a Hecke operator as a graph with weighted edges that encodes the action of a Hecke operator on automorphic forms. This definition applies to every Hecke operator of $\mathrm{PGL}_{2}(\mathbb{A})$ over a global field. We collect first properties of these graphs and describe how the algebraic structure of the Hecke algebra is reflected in dependencies between the graphs. In Section 2, we describe the graph $\mathscr{G}_{x}$ of the unramified Hecke operators $\Phi_{x}$ (which correspond to the local Hecke operators $T_{x}$ as introduced above) in terms of coset representatives. In Section 3, we make the 
connection to Bruhat-Tits trees precise: Each component of $\mathscr{G}_{x}$ is a quotient of $\mathscr{T}_{x}$ by a certain subgroup of $\mathrm{PGL}_{2}\left(F_{x}\right)$, and the components of $\mathscr{G}_{x}$ are counted by the 2 -torsion of the class group of $\mathrm{O}_{F}^{x}$. In Section 4 , we associate to each vertex of $\varphi_{x}$ a coset in $\mathrm{Cl} F / 2 \mathrm{Cl} F$ where $\mathrm{Cl} F$ is the divisor class group of $F$. We describe how these labels are distributed in $\varphi_{x}$ in dependence of $x$.

In Section 5, we give the vertices and edges of $\varphi_{x}$ a geometric meaning following ideas connected to the geometric Langlands program. Namely, the vertices correspond to the isomorphism classes of $\mathbb{P}^{1}$-bundles on the smooth projective curve $X$ with function field $F$, and the edges correspond to certain exact sequences of sheaves on $X$. In Section 6, we distinguish three classes of rank 2 bundles: those that decompose into a sum of two line bundles, those that are the trace of a line bundle over the quadratic constant extension $X^{\prime}$ of $X$ and those that are geometrically indecomposable. This divides the vertices of $\mathscr{G}_{x}$ into three subclasses $\mathbb{P B u n}_{2}^{\text {dec }} X, \mathbb{P B u n}{ }_{2}^{\text {tr }} X$ and $\mathbb{P B u n}{ }_{2}^{\text {gi }} X$. The former two sets of vertices have a simple description in terms of the divisor class groups of $X$ and $X^{\prime}$.

In Section 7, we introduce the integer valued invariant $\delta$ on the set of vertices, which is closely connected to reduction theory of rank 2 bundles. This helps us to refine our view on the vertices: $\mathbb{P B u n}{ }_{2}^{\text {tr }} X$ and $\mathbb{P B u n}_{2}^{\text {gi }} X$ are contained in the finite set of vertices $v$ with $\delta(v) \leq 2 g_{X}-2$, where $g_{X}$ is the genus of $X$. In Section 8, we describe the edges between vertices: $\mathscr{G}_{x}$ decomposes into a finite graph, which depends heavily on the arithmetic of $F$, and class-number-many cusps, which are infinite weighted subgraphs of the form

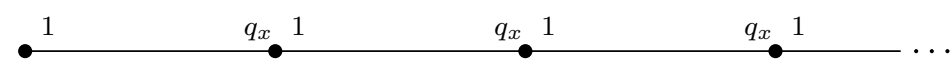

We conclude with a summary of results on $\mathscr{G}_{x}$ and illustrate them in Figure 8a.

In Section 9, we explain how abstract properties of unramified automorphic forms - namely, the compact support of cusp forms and eigenvalue equations for Eisenstein series - lead to an explicit description of them as functions on the vertices of the graphs $\mathscr{G}_{x}$. In Section 10, we show that the spaces of functions on Vert $G_{x}$ that satisfy the cuspidal or toroidal conditions, respectively, are finite dimensional. In particular, these spaces of functions contain only automorphic forms.

In the appendix, we will give a series of examples for a rational function field: $\mathscr{G}_{x}$ for $\operatorname{deg} x \leq 5$, the graphs of $\Phi_{x}^{2}$ and $\Phi_{x}^{3}$ for $\operatorname{deg} x=1$ and the graphs of two ramified Hecke operators. We give short explanations on how to calculate these examples. 


\section{Definitions}

In this section, we set up our notation and introduce the notion of a graph of a Hecke operator. We collect first properties of these graphs and describe how the algebraic structure of the Hecke algebra is reflected in dependencies between the graphs of different Hecke operators.

1.1. Let $q$ be a prime power and $F$ be the function field of a smooth projective curve $X$ over $\mathbb{F}_{q}$. Let $\|X\|$ be the set of closed points of $X$, which we identify with the set of places of $F$. We denote by $F_{x}$ the completion of $F$ at $x \in\|X\|$ and by $O_{x}$ the integers of $F_{x}$. We choose a uniformizer $\pi_{x} \in F$ for every place $x$. Let $\kappa_{x}=O_{x} /\left(\pi_{x}\right)$ be the residue field. Let $\operatorname{deg} x$ be the degree of $x$ and let $q_{x}=q^{\operatorname{deg} x}$ be the cardinality of $\kappa_{x}$. We denote by $\|\cdot\|_{x}$ the absolute value on $F_{x}$ and $F$, respectively, such that $\left\|\pi_{x}\right\|_{x}=q_{x}^{-1}$.

Let $\mathbb{A}$ be the adèle ring of $F$ and $\mathbb{A}^{\times}$the idèle group. Put $O_{\mathbb{A}}=\prod O_{x}$, where the product is taken over all places $x$ of $F$. The idèle norm is the quasicharacter $\|\cdot\|: \mathbb{A}^{\times} \rightarrow \mathbb{C}^{\times}$that sends an idèle $\left(a_{x}\right) \in \mathbb{A}^{\times}$to the product $\prod\left\|a_{x}\right\|_{x}$ over all local norms. By the product formula, this defines a quasicharacter on the idèle class group $A^{\times} / F^{\times}$.

We think of $F_{x}$ being embedded into the adèle ring $A$ by sending an element $a$ of $F_{x}$ to the adèle $\left(a_{y}\right)$ with $a_{x}=a$ and $a_{y}=0$ for $y \neq x$. It being not quite compatible with this embedding, we think of the unit group $F_{x}^{\times}$as a subgroup of the idèle group $\mathbb{A}^{\times}$by sending an element $b$ of $F_{x}^{\times}$to the idèle $\left(b_{y}\right)$ with $b_{x}=b$ and $b_{y}=1$ for $y \neq x$. We will explain, in case of ambiguity, which of these embeddings we use.

Let $G=\mathrm{PGL}_{2}$. Following the habit of literature about automorphic forms, we will often write $G_{\mathbb{A}}$ instead of $G(\mathbb{A})$ for the group of adelic points and $G_{F}$ instead of $G(F)$ for the group of $F$-valued points, et cetera. Note that $G_{\AA}$ comes together with an adelic topology that turns $G_{\AA}$ into a locally compact group. Let $K=G_{\mathscr{C}_{\mathrm{A}}}$ be the standard maximal compact open subgroup of $G_{\AA}$. We fix the Haar measure on $G_{A}$ for which vol $K=1$.

The Hecke algebra $\mathscr{H}$ for $G_{\mathbb{A}}$ is the complex vector space of all compactly supported locally constant functions $\Phi: G_{\mathbb{A}} \rightarrow \mathbb{C}$ together with the convolution product

$$
\Phi_{1} * \Phi_{2}: g \mapsto \int_{G_{\AA}} \Phi_{1}\left(g h^{-1}\right) \Phi_{2}(h) d h .
$$

A Hecke operator $\Phi \in \mathscr{H}$ acts on the space $\mathscr{V}=C^{0}\left(G_{F} \backslash G_{A}\right)$ of continuous functions $f: G_{F} \backslash G_{\AA} \rightarrow \mathbb{C}$ by the formula

$$
\Phi(f)(g)=\int_{G_{\AA}} \Phi(h) f(g h) d h .
$$


Let $K^{\prime}$ be a compact open subgroup of $G_{\AA}$. Then we denote by $\mathscr{H}_{K^{\prime}}$ the subalgebra of $\mathscr{H}$ that consists of all bi- $K^{\prime}$-invariant functions. The action above restricts to an action of $\mathcal{H}_{K^{\prime}}$ on $\mathscr{V} K^{\prime}$, the space of right $K^{\prime}$-invariant functions.

Lemma 1.2. For every $K^{\prime}$ and every $\Phi \in \mathscr{H}_{K^{\prime}}$, there are $h_{1}, \ldots, h_{r} \in G_{\mathbb{A}}$ and $m_{1}, \ldots, m_{r} \in \mathbb{C}$ for some integer $r$ such that for all $g \in G_{\mathbb{A}}$ and all $f \in \mathscr{V}^{K^{\prime}}$,

$$
\Phi(f)(g)=\sum_{i=1}^{r} m_{i} \cdot f\left(g h_{i}\right) .
$$

Proof. Since $\Phi$ is $K^{\prime}$-biinvariant and compactly supported, it is a finite linear combination of characteristic functions on double cosets of the form $K^{\prime} h K^{\prime}$ with $h \in G_{\mathrm{A}}$. So we may reduce the proof to the case $\Phi=\operatorname{char}_{K^{\prime} h K^{\prime}}$. Again, since $K^{\prime} h K^{\prime}$ is compact, it equals the union of a finite number of pairwise distinct cosets $h_{1} K^{\prime}, \ldots, h_{r} K^{\prime}$, and thus, for arbitrary $g \in G_{\mathbb{A}}$,

$$
\begin{aligned}
\int_{G_{\AA}} \operatorname{char}_{K^{\prime} h K^{\prime}}\left(h^{\prime}\right) f\left(g h^{\prime}\right) d h^{\prime} & =\sum_{i=1}^{r} \int_{G_{\mathbb{A}}} \operatorname{char}_{h_{i} K^{\prime}}\left(h^{\prime}\right) f\left(g h^{\prime}\right) d h \\
& =\sum_{i=1}^{r} \operatorname{vol}\left(K^{\prime}\right) f\left(g h_{i}\right) .
\end{aligned}
$$

We will write $[g] \in G_{F} \backslash G_{\AA} / K^{\prime}$ for the class that is represented by $g \in G_{\AA}$. Other cosets will also occur in this paper, but it will be clear from the context what kind of class the square brackets relate to.

Proposition 1.3. For all $\Phi \in \mathscr{H}_{K^{\prime}}$ and $[g] \in G_{F} \backslash G_{\mathbb{A}} / K^{\prime}$, there is a unique set of pairwise distinct classes $\left[g_{i}\right] \in G_{F} \backslash G_{\mathbb{A}} / K^{\prime}$ and numbers $m_{i} \in \mathbb{C}^{\times}$, for $1 \leq i \leq r$, such that for all $f \in \mathscr{V}^{K^{\prime}}$,

$$
\Phi(f)(g)=\sum_{i=1}^{r} m_{i} f\left(g_{i}\right) .
$$

Proof. Uniqueness is clear, and existence follows from Lemma 1.2 after we have taken care of putting together values of $f$ in same classes of $G_{F} \backslash G_{\mathbb{A}} / K^{\prime}$ and excluding the zero terms.

Definition 1.4. With the notation of the preceding proposition we define

$$
\mathcal{U}_{\Phi, K^{\prime}}([g])=\left\{\left([g],\left[g_{i}\right], m_{i}\right)\right\}_{i=1, \ldots, r} .
$$

The classes $\left[g_{i}\right]$ are called the $\Phi$-neighbors of $[g]$ (relative to $K^{\prime}$ ), and the $m_{i}$ are called their weights.

The graph $\mathscr{G}_{\Phi, K^{\prime}}$ of $\Phi$ (relative to $K^{\prime}$ ) consists of vertices

$$
\operatorname{Vert} \varphi_{\Phi, K^{\prime}}=G_{F} \backslash G_{\mathrm{A}} / K^{\prime}
$$


and oriented weighted edges

$$
\text { Edge } \mathscr{G}_{\Phi, K^{\prime}}=\bigcup_{v \in \operatorname{Vert} \varphi_{\Phi, K^{\prime}}} \boldsymbol{U}_{\Phi, K^{\prime}}(v)
$$

Remark 1.5. The usual notation for an edge in a graph with weighted edges consists of pairs that code the origin and the terminus, and an additional function on the set of edges that gives the weight. For our purposes, it is more convenient to replace the set of edges by the graph of the weight function and to call the resulting triples that consist of origin, terminus and the weight the edges of $\mathscr{G}_{\Phi, K^{\prime}}$.

1.6. We make the following drawing conventions to illustrate the graph of a Hecke operator: vertices are represented by labeled dots, and an edge $\left(v, v^{\prime}, m\right)$ together with its origin $v$ and its terminus $v^{\prime}$ is drawn as

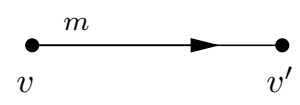

If there is precisely one edge from $v$ to $v^{\prime}$ and precisely one from $v^{\prime}$ to $v$, which we call the inverse edge, we draw

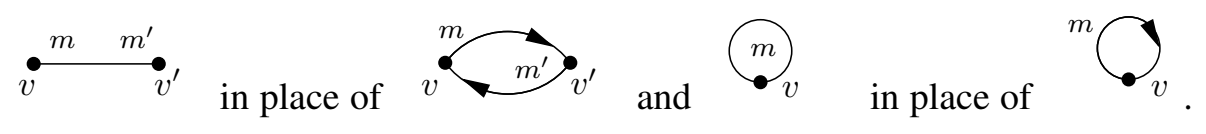

There are various examples for rational function fields in the appendix, and in [Lorscheid 2012], one finds graphs of Hecke operators for elliptic function fields.

1.7. We collect some properties that follow immediately from the definition of a graph of a Hecke operator $\Phi$. For $f \in \mathscr{V}^{K^{\prime}}$ and $[g] \in G_{F} \backslash G_{\mathbb{A}} / K^{\prime}$, we have

$$
\Phi(f)(g)=\sum_{\substack{\left[[g],\left[g^{\prime}\right], m^{\prime}\right) \\ \in \operatorname{Edge} \mathscr{S}_{\Phi, K^{\prime}}}} m^{\prime} f\left(g^{\prime}\right) .
$$

Hence one can read off the action of a Hecke operator on $f \in \mathscr{V}^{K^{\prime}}$ from the illustration of the graph

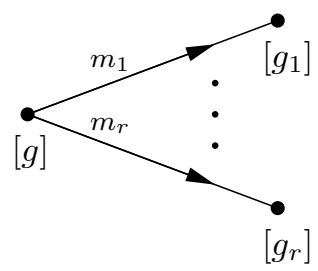

Since $\mathscr{H}=\bigcup \mathscr{H}_{K^{\prime}}$, with $K^{\prime}$ running over all compact opens in $G_{\mathrm{A}}$, the notion of the graph of a Hecke operator applies to any $\Phi \in \mathcal{H}$. The set of vertices of the 
graph of a Hecke operator $\Phi \in \mathscr{H}_{K^{\prime}}$ only depends on $K^{\prime}$, and only the edges depend on the particular chosen $\Phi$. There is at most one edge for each pair of vertices and each direction, and the weight of an edge is always nonzero. Each vertex is connected with only finitely many other vertices.

The algebra structure of $\mathscr{H}_{K^{\prime}}$ has the following implications on the structure of the set of edges (with the convention that the empty sum is defined as 0 ). For the zero element $0 \in \mathscr{H}_{K^{\prime}}$, the multiplicative unit $1 \in \mathscr{H}_{K^{\prime}}$, and arbitrary $\Phi_{1}, \Phi_{2} \in \mathscr{H}_{K^{\prime}}$ and $r \in \mathbb{C}^{\times}$, we obtain

$$
\begin{aligned}
\text { Edge } \mathscr{G}_{0, K^{\prime}} & =\varnothing, \\
\text { Edge } \mathscr{G}_{1, K^{\prime}} & =\{(v, v, 1)\}_{v \in \operatorname{Vert} \varphi_{1, K^{\prime}},} \\
\text { Edge } \mathscr{G}_{\Phi_{1}+\Phi_{2}, K^{\prime}} & =\left\{\left(v, v^{\prime}, m\right) \mid m=\sum_{\substack{\left(v, v^{\prime}, m^{\prime}\right) \\
\in \operatorname{Edge} \varphi_{\Phi_{1}, K^{\prime}}}} m^{\prime}+\sum_{\begin{array}{c}
\left(v, v^{\prime}, m^{\prime \prime}\right) \in \\
\operatorname{Edge} \varphi_{\Phi_{2}, K^{\prime}}
\end{array}} m^{\prime \prime} \neq 0\right\}, \\
\text { Edge } \mathscr{G}_{r} \Phi_{1}, K^{\prime} & =\left\{\left(v, v^{\prime}, r m\right) \mid\left(v, v^{\prime}, m\right) \in \operatorname{Edge} \mathscr{G}_{\Phi_{1}, K^{\prime}}\right\}, \\
\text { Edge } \mathscr{G}_{\Phi_{1} * \Phi_{2}, K^{\prime}} & =\left\{\left(v, v^{\prime}, m\right) \mid m=\sum_{\substack{\left(v, v^{\prime \prime}, m^{\prime}\right) \in \operatorname{Edge} \varphi_{\Phi_{1}, K^{\prime}},\left(v^{\prime \prime}, v^{\prime}, m^{\prime \prime}\right) \in \operatorname{Edge} \varphi_{\Phi_{2}, K^{\prime}}}} m^{\prime} \cdot m^{\prime \prime} \neq 0\right\} .
\end{aligned}
$$

If $K^{\prime \prime}<K^{\prime}$ and $\Phi \in \mathscr{H}_{K^{\prime}}$, then also $\Phi \in \mathscr{H}_{K^{\prime \prime}}$. This implies that we have a canonical map $P: \mathscr{G}_{\Phi, K^{\prime \prime}} \rightarrow \mathscr{G}_{\Phi, K^{\prime}}$, which is given by

$$
\begin{aligned}
\operatorname{Vert} \mathscr{G}_{\Phi, K^{\prime \prime}}= & G_{F} \backslash G_{\mathbb{A}} / K^{\prime \prime} \stackrel{P}{\rightarrow} G_{F} \backslash G_{\AA} / K^{\prime}=\operatorname{Vert} \mathscr{G}_{\Phi, K^{\prime}}, \\
& \quad \operatorname{Edge} \mathscr{G}_{\Phi, K^{\prime \prime}} \stackrel{P}{\rightarrow} \operatorname{Edge} \mathscr{G}_{\Phi, K^{\prime}}, \quad\left(v, v^{\prime}, m^{\prime}\right) \mapsto\left(P(v), P\left(v^{\prime}\right), m^{\prime}\right) .
\end{aligned}
$$

1.8. One can also collect the data of $\varphi_{\Phi, K^{\prime}}$ in an infinite-dimensional matrix $M_{\Phi, K^{\prime}}$, which we call the matrix associated with $\mathscr{G}_{\Phi, K^{\prime}}$, by putting $\left(M_{\Phi, K^{\prime}}\right)_{v^{\prime}, v}=m$ if $\left(v, v^{\prime}, m\right) \in \operatorname{Edge} \mathscr{G}_{\Phi, K^{\prime}}$, and $\left(M_{\Phi, K^{\prime}}\right)_{v^{\prime}, v}=0$ otherwise. Thus each row and each column has only finitely many nonvanishing entries.

The properties of the last paragraph imply the following:

$$
\begin{array}{lrl}
M_{0, K^{\prime}}=0, \text { the zero matrix, } & M_{\Phi_{1}+\Phi_{2}, K^{\prime}}=M_{\Phi_{1}, K^{\prime}}+M_{\Phi_{2}, K^{\prime}}, \\
M_{1, K^{\prime}}=1, \text { the identity matrix, } & M_{r \Phi_{1}, K^{\prime}}=r M_{\Phi_{1}, K^{\prime}}, \\
M_{\Phi_{1} * \Phi_{2}, K^{\prime}} & =M_{\Phi_{2}, K^{\prime}} M_{\Phi_{1}, K^{\prime}} .
\end{array}
$$

Let $\mathscr{F}\left(K^{\prime}\right) \subset \mathscr{H}_{K^{\prime}}$ be the ideal of operators that act trivially on $\mathscr{V}^{K^{\prime}}$. Then we may regard $\mathscr{H}_{K^{\prime}} / \mathscr{F}\left(K^{\prime}\right)$ as a subalgebra of the algebra of $\mathbb{C}$-linear maps

$$
\bigoplus_{G_{F} \backslash G_{\AA} / K^{\prime}} \mathbb{C} \rightarrow \bigoplus_{G_{F} \backslash G_{\AA} / K^{\prime}} \mathbb{C} .
$$




\section{Unramified Hecke operators}

From now on we will restrict ourselves to unramified Hecke operators, which means elements in $\mathscr{H}_{K}$. In particular, we will investigate the graphs $\mathscr{G}_{x}$ of certain generators $\Phi_{x}$ of $\mathscr{H}_{K}$ in more detail.

2.1. Consider the uniformizers $\pi_{x} \in F$ as idèles via the embedding $F^{\times} \subset F_{x}^{\times} \subset \mathbb{A}^{\times}$ and define for every place $x$ the unramified Hecke operator $\Phi_{x}$ as the characteristic function of $K\left(\begin{array}{c}\pi_{x} \\ { }_{1}\end{array}\right) K$. It is well known that $\mathscr{H}_{K} \simeq \mathbb{C}\left[\Phi_{x}\right]_{x \in\|X\|}$ as an algebra, which means, in particular, that $\mathscr{H}_{K}$ is commutative. By the relations from Section 1.7, it is enough to know the graphs of generators to determine all graphs of unramified Hecke operators. We use the shorthand notation $\mathscr{G}_{x}$ for the graph $\varphi_{\Phi_{x}, K}$, and $\boldsymbol{U}_{x}(v)$ for the $\Phi_{x}$-neighbors $U_{\Phi_{x}, K}(v)$ of $v$.

We introduce the lower $x$ convention that says that a lower index $x$ on an algebraic group defined over the adèles of $F$ will consist of only the component at $x$ of the adelic points, for example, $G_{x}=G_{F_{x}}$. Analogously, we put $K_{x}=G_{\mathscr{C}_{x}}$.

The upper $x$ convention means that an upper index $x$ on an algebraic group defined over the adèles of $F$ will consist of all components except for the one at $x$. In particular, we first define $\mathbb{A}^{x}=\prod_{y \neq x}^{\prime} F_{y}$, the restricted product relative to $\mathcal{O}^{x}=\prod_{y \neq x} \mathrm{O}_{y}$ over all places $y$ that do not equal $x$. Another example is $G^{x}=G_{\mathbb{A}^{x}}$. We put $K^{x}=G_{0^{x}}$.

2.2. We embed $\kappa_{x}$ via $\kappa_{x} \subset F_{x} \subset \mathbb{A}$; thus an element $b \in \kappa_{x}$ will be considered as the adèle whose component at $x$ is $b$ and whose other components are 0 . Let $\mathbb{P}^{1}$ be the projective line. Define, for $w \in \mathbb{P}^{1}\left(\kappa_{x}\right)$,

$$
\xi_{w}=\left(\begin{array}{ll}
\pi_{x} & b \\
& 1
\end{array}\right) \quad \text { if } w=[1: b] \quad \text { and } \quad \xi_{w}=\left(\begin{array}{ll}
1 & \\
& \pi_{x}
\end{array}\right) \quad \text { if } w=[0: 1] .
$$

It is well known (see [Gelbart 1975, Lemma 3.7]) that the domain of $\Phi_{x}$ can be described as

$$
K\left(\begin{array}{ll}
\pi_{x} & \\
& 1
\end{array}\right) K=\bigsqcup_{w \in \mathbb{P}^{1}\left(\kappa_{x}\right)} \xi_{w} K .
$$

Consequently the weights of edges in $\mathscr{G}_{x}$ are positive integers (recall that vol $K=1$ ). We shall also refer to the weights as the multiplicity of a $\Phi_{x}$-neighbor. The above implies the following.

Proposition 2.3. The $\Phi_{x}$-neighbors of $[g]$ are the classes $\left[g \xi_{w}\right]$ with $\xi_{w}$ as in the previous paragraph, and the multiplicity of an edge from $[g]$ to $\left[g^{\prime}\right]$ equals the number of $w \in \mathbb{P}^{1}\left(\kappa_{x}\right)$ such that $\left[g \xi_{w}\right]=\left[g^{\prime}\right]$. The multiplicities of the edges originating in $[g]$ sum up to $\# \mathbb{P}^{1}\left(\kappa_{x}\right)=q_{x}+1$. 


\section{Connection with Bruhat-Tits trees}

Fix a place $x$. In this section we construct maps from Bruhat-Tits trees to $\mathscr{G}_{x}$. This will enable us to determine the components of $\mathscr{G}_{x}$.

Definition 3.1. The Bruhat-Tits tree $\mathscr{T}_{x}$ for $F_{x}$ is the (unweighted) graph with vertices Vert $\mathscr{T}_{x}=G_{x} / K_{x}$ and edges

$$
\text { Edge } \mathscr{T}_{x}=\left\{\left([g],\left[g^{\prime}\right]\right) \mid \exists w \in \mathbb{P}^{1}\left(\kappa_{x}\right), g \equiv g^{\prime} \xi_{w}\left(\bmod K_{x}\right)\right\} .
$$

3.2. Consider $G_{x}$ to be embedded in $G_{\AA}$ as the component at $x$. For each $h \in G_{\AA}$, we define a map $\Psi_{x, h}: \mathscr{T}_{x} \rightarrow \mathscr{G}_{x}$ by

$$
\begin{aligned}
\operatorname{Vert} \mathscr{T}_{x}=G_{x} / K_{x} & \rightarrow G_{F} \backslash G_{A} / K=\operatorname{Vert} \mathscr{G}_{x}, & \text { Edge } \mathscr{T}_{x} & \rightarrow \text { Edge } \mathscr{G}_{x}, \\
{[g] } & \mapsto[h g], & \left([g],\left[g^{\prime}\right]\right) & \mapsto\left([h g],\left[h g^{\prime}\right], m\right),
\end{aligned}
$$

with $m$ being the number of vertices $\left[g^{\prime \prime}\right]$ that are adjacent to $[g]$ in $\mathscr{T}_{x}$ such that $\Psi_{x, h}\left(\left[g^{\prime \prime}\right]\right)=\Psi_{x, h}\left(\left[g^{\prime}\right]\right)$.

By Proposition 2.3 and the definition of a Bruhat-Tits tree, $\Psi_{x, h}$ is well-defined and locally surjective, that is, it is locally surjective as a map between the associated simplicial complexes of $\mathscr{T}_{x}$ and $\mathscr{G}_{x}$ with suppressed weights.

Since Bruhat-Tits trees are indeed trees [Serre 2003, II.1, Theorem 1], hence in particular connected, the image of each $\Psi_{x, h}$ is precisely one component of $\varphi_{x}$, that is, a subgraph that corresponds to a connected component of the associated simplicial complex.

Every edge of the Bruhat-Tits tree has an inverse edge, which implies the analogous statement for the graphs $\mathscr{G}_{x}$. Namely, if $\left(v, v^{\prime}, m\right) \in$ Edge $\mathscr{G}_{x}$, then there is an $m^{\prime} \in \mathbb{C}^{\times}$such that $\left(v^{\prime}, v, m^{\prime}\right) \in \operatorname{Edge} \mathscr{G}_{x}$.

Remark 3.3. This symmetry of edges is a property that is particular to unramified Hecke operators for $G=\mathrm{PGL}_{2}$. In case of ramification, the symmetry is broken; see Example A.7.

3.4. The algebraic group $\mathrm{SL}_{2}$ has the strong approximation property, that is, for every place $x, \mathrm{SL}_{2} F$ is a dense subset of $\mathrm{SL}_{2} A^{x}$ with respect to the adelic topology. See [Bourbaki 1965, §2, nombre 4; Kneser 1966; Moore 1968, Chapter IV, Lemma 13.1; Margulis 1977; Prasad 1977] for the development of the strong approximation results and their generalizations to all simple groups. See also [Laumon 1997, Theorem E.2.1] for a proof. We explain what implication this has on $\mathrm{PGL}_{2}$. More detail for the outline in this paragraph can be found in [van der Put and Reversat 1997, (2.1.3)].

Let $x$ be a place of degree $d$. In accordance to the upper $x$ convention, let $\mathcal{O}^{x}=\prod_{y \neq x} \mathcal{O}_{y}$. As a consequence of the strong approximation property of $\mathrm{SL}_{n}$, the 
determinant map on $\mathrm{GL}_{2}$ induces a bijection on double cosets:

$$
\mathrm{GL}_{2}(F) \backslash \mathrm{GL}_{2}\left(\mathbb{A}^{x}\right) / \mathrm{GL}_{2}\left(\mathbb{O}^{x}\right) \stackrel{\operatorname{det}}{\longrightarrow} F^{\times} \backslash\left(\mathbb{A}^{x}\right)^{\times} /\left(\mathbb{O}^{x}\right)^{\times} .
$$

The quotient group $F^{\times} \backslash\left(\mathbb{A}^{x}\right)^{\times} /\left(\mathcal{O}^{x}\right)^{\times}$is nothing else but the ideal class group $\mathrm{ClO} \mathcal{O}_{F}^{x}$ of the integers $\mathcal{O}_{F}^{x}=\mathcal{O}^{x} \cap F$ coprime to $x$. Let $\mathrm{Cl} F=F^{\times} \backslash \mathbb{A}^{\times} / \mathcal{O}_{A}^{\times}$be the divisor class group of $F$ and $\mathrm{Cl}^{0} F=\{[a] \in \mathrm{Cl} F \mid \operatorname{deg} a=0\}$ be the ideal class group. Then we have bijections

$$
\mathrm{GL}_{2}(F) \backslash \mathrm{GL}_{2}\left(\mathbb{A}^{x}\right) / \mathrm{GL}_{2}\left(\mathbb{O}^{x}\right) \simeq F^{\times} \backslash\left(\mathbb{A}^{x}\right)^{\times} /\left(\mathcal{O}^{x}\right)^{\times} \simeq \mathrm{ClO}_{F}^{x} \simeq \mathrm{Cl}^{0} F \times \mathbb{Z} / d \mathbb{Z} .
$$

Let $S \subset \mathrm{GL}_{2}\left(\mathbb{A}^{x}\right)$ be a set of representatives for $\mathrm{GL}_{2}(F) \backslash \mathrm{GL}_{2}\left(\mathbb{A}^{x}\right) / \mathrm{GL}_{2}\left(\mathcal{O}^{x}\right)$. Then for every $g=g^{x} g_{x} \in \mathrm{GL}_{2}(\mathbb{A})$ (with $g^{x} \in \mathrm{GL}_{2}\left(\mathbb{A}^{x}\right)$ and $g_{x} \in \mathrm{GL}_{2}\left(F_{x}\right)$ ), there are $s \in S, \gamma \in \mathrm{GL}_{2}(F)$ and $k \in \mathrm{GL}_{2}\left(\mathbb{O}^{x}\right)$ such that $g=\gamma s k \tilde{g}_{x}$, where $\gamma s k$ equals $g$ in all components $z \neq x$ and $\tilde{g}_{x}=\gamma^{-1} g_{x}$. The condition [det $\left.s\right]=\left[\operatorname{det} g^{x}\right]$ as cosets in $F^{\times} \backslash\left(\mathbb{A}^{x}\right)^{\times} /\left(\mathbb{O}^{x}\right)^{\times}$implies that $s \in S$ is uniquely determined by $g^{x}$. Let $Z$ be the center of $\mathrm{GL}_{2}$. Then

$$
\begin{aligned}
\mathrm{GL}_{2}(\mathbb{A}) / \mathrm{GL}_{2}\left(\mathrm{O}_{\mathbb{A}}\right) Z_{x} & =\mathrm{GL}_{2}\left(\mathbb{A}^{x}\right) / \mathrm{GL}_{2}\left(\mathbb{O}^{x}\right) \times G_{x} / K_{x} \\
& =\mathrm{GL}_{2}\left(\mathbb{A}^{x}\right) / \mathrm{GL}_{2}\left(\mathbb{O}^{x}\right) \times \operatorname{Vert} \mathscr{T}_{x} .
\end{aligned}
$$

Define $\Gamma_{s}=\mathrm{GL}_{2}(F) \cap s \mathrm{GL}_{2}\left(\mathbb{O}^{x}\right) s^{-1}$. Then we obtain the following; see [van der Put and Reversat 1997, (2.1.3)].

Proposition 3.5. The decomposition $g=\gamma s k \tilde{g}_{x}$ induces a bijective map

$$
\mathrm{GL}_{2}(F) \backslash \mathrm{GL}_{2}\left(\mathbb{A}^{x}\right) / \mathrm{GL}_{2}\left(\mathcal{O}_{\mathbb{A}}\right) Z_{x} \rightarrow \bigsqcup_{s \in S} \Gamma_{s} \backslash \operatorname{Vert} \mathscr{T}_{x}, \quad[g] \mapsto\left(s,\left[\tilde{g}_{x}\right]\right) .
$$

Its inverse is obtained by joining the components $s \in \mathrm{GL}_{2}\left(\mathbb{A}^{x}\right)$ and $\tilde{g}_{x} \in G_{x}$.

Remark 3.6. On the right side of the bijection in Proposition 3.5, we have a finite union of quotients of the form $\Gamma_{s} \backslash$ Vert $\mathscr{T}_{x}$. If $s$ is the identity element $e$, then $\Gamma=\Gamma_{e}=\mathrm{GL}_{2}\left(\mathrm{O}_{F}^{x}\right)$ is an arithmetic group of the form considered in [Serre 2003, II.2.3]. For general $s, \mathrm{I}$ am not aware of any results about $\Gamma_{s} \backslash$ Vert $\mathscr{T}_{x}$.

3.7. So far, we have only divided out the action of the $x$-component $Z_{x}$ of the center. We still have to consider the action of $Z^{x}$. The image of $Z^{x}$ under the determinant det $: \mathrm{GL}_{2}\left(\mathbb{A}^{x}\right) \rightarrow \mathrm{ClO}_{F}^{x}$ is $2 \mathrm{ClO}_{F}^{x}$. Thus we obtain a bijection

$$
Z^{x} \mathrm{GL}_{2}(F) \backslash \mathrm{GL}_{2}\left(\mathbb{A}^{x}\right) / \mathrm{GL}_{2}\left(0^{x}\right) \stackrel{\operatorname{det}}{\rightarrow} \mathrm{Cl} 0_{F}^{x} / 2 \mathrm{ClO}_{F}^{x}
$$

The double quotient on the left side can be identified with $G_{F} \backslash G^{x} / K^{x}$. Let $J=\left\{z \in Z^{x} \mid \operatorname{det} z=0 \in \mathrm{ClO}_{F}^{x}\right\}$ be the kernel of the restriction det : $Z^{x} \rightarrow \mathrm{ClO}_{F}^{x}$ and define $\tilde{\Gamma}_{s}=\mathrm{GL}_{2}(F) \cap J_{s} \mathrm{GL}_{2}\left(\mathcal{O}^{x}\right) s^{-1}$. If we let $S^{\prime} \subset S$ be a set of representatives 
for $\mathrm{ClO}_{F}^{x} / 2 \mathrm{ClO}_{F}^{x}$ (with respect to the determinant map), and $h_{2}=\#(\mathrm{Cl} F)[2]$ the cardinality of the 2-torsion, then we obtain:

Proposition 3.8. The decomposition $g=\gamma s k \tilde{g}_{x}$ induces a bijective map

$$
G_{F} \backslash G_{\AA} / K \rightarrow \bigsqcup_{s \in S^{\prime}} \tilde{\Gamma}_{s} \backslash \operatorname{Vert} \mathscr{T}_{x} .
$$

The inverse maps an element $\left(s,\left[\tilde{g}_{x}\right]\right)$ to the class of the adelic matrix with components $s \in G^{x}$ and $\tilde{g}_{x} \in G_{x}$. The number of components of $\mathscr{G}_{x}$ equals

$$
\#\left(\mathrm{ClO}_{F}^{x} / 2 \mathrm{ClO}_{F}^{x}\right)=\#\left(\mathrm{Cl} O_{F}^{x}\right)[2]= \begin{cases}h_{2} & \text { if } \operatorname{deg} x \text { is odd }, \\ 2 h_{2} & \text { if } \operatorname{deg} x \text { is even } .\end{cases}
$$

Proof. Everything follows from Proposition 3.5 and Section 3.7 except for the two equalities in the last line. The former equality follows from the general fact that one has \# $\operatorname{ker} f=\#(G / \operatorname{im} f)$ for a homomorphism $f$ acting on a finite group $G$ (in our case $f$ is the multiplication by 2 ). The latter equality follows immediately from the observation $\mathrm{Cl}_{F}^{x} \simeq \mathrm{Cl}^{0} F \times \mathbb{Z} / d \mathbb{Z}$, where $d=\operatorname{deg} x$.

\section{A vertex labeling}

In this section, we associate to each vertex of $\varphi_{x}$ an element of $\mathrm{Cl} F / 2 \mathrm{Cl} F$ and determine how these labels are distributed over the components of $\mathscr{G}_{x}$.

4.1. Let $\mathscr{2}_{\mathbb{A}}=\left\langle a^{2} \mid a \in \mathbb{A}^{\times}\right\rangle$be the subgroup of squares. We look once more at the determinant map

$$
\operatorname{Vert} \varphi_{x}=G_{F} \backslash G_{\mathbb{A}} / K \stackrel{\operatorname{det}}{\longrightarrow} F^{\times} \backslash \mathbb{A}^{\times} / \mathbb{O}_{\mathbb{A}^{\times}} \mathscr{Q}_{\mathbb{A}} \simeq \mathrm{Cl} F / 2 \mathrm{Cl} F .
$$

This map assigns to every vertex in $\mathscr{G}_{x}$ a label in $\mathrm{Cl} F / 2 \mathrm{Cl} F$, which has $2 h_{2}$ elements, where $h_{2}=\#(\mathrm{Cl} F)[2]$, for the same reason as used in the proof of Proposition 3.8.

Proposition 4.2. If the prime divisor $x$ is a square in the divisor class group, then all vertices in the same component of $G_{x}$ have the same label, and there are $2 h_{2}$ components, each of which has a different label. Otherwise, the vertices of each component have one of two labels that differ by $x$ in $\mathrm{Cl} F / 2 \mathrm{Cl} F$, and two adjacent vertices have different labels, so each connected component is bipartite.

Proof. First of all, observe that each label is realized, since if we represent a label by some idèle $a$, then the vertex represented by $\left({ }^{a}{ }_{1}\right)$ has this label.

Let $2_{x}=\left\langle b^{2} \mid b \in F_{x}^{\times}\right\rangle$and $\mathrm{Cl} F_{x}=F_{x}^{\times} / \mathcal{O}_{x}^{\times}$, a group isomorphic to $\mathbb{Z}$. For the Bruhat-Tits tree $\mathscr{T}_{x}$, the determinant map

$$
\operatorname{Vert} \mathscr{T}_{x}=G_{x} / K_{x} \stackrel{\text { det }}{\longrightarrow} F_{x}^{\times} / \mathbb{O}_{x}^{\times} \mathscr{Q}_{x} \simeq \mathrm{Cl} F_{x} / 2 \mathrm{Cl} F_{x} \simeq \mathbb{Z} / 2 \mathbb{Z}
$$


defines a labeling of the vertices, and the two classes of $F_{x}^{\times} / \mathcal{O}_{x}^{\times} \mathscr{2}_{x}$ are represented by 1 and $\pi_{x}$. Two adjacent vertices have the different labels since for $g \in G_{x}$ and $\xi_{w}$ as in Definition 3.1, $\operatorname{det}\left(g \xi_{w}\right)=\pi_{x} \operatorname{det} g$ represents a class different from det $g$ in $\operatorname{Vert} \mathscr{T}_{x}$.

Define for $a \in \mathbb{A}^{\times}$a map $\psi_{x, a}: F_{x}^{\times} / \mathscr{O}_{x}^{\times} \mathscr{2}_{x} \rightarrow F^{\times} \backslash \mathbb{A}^{\times} / \mathbb{O}_{\mathbb{A}}^{\times} \mathscr{Q}_{\mathbb{A}}$ by $\psi_{x, a}([b])=[a b]$, where $b$ is viewed as the idèle concentrated in $x$. For every $h \in G_{\mathbb{A}}$ we obtain a commutative diagram

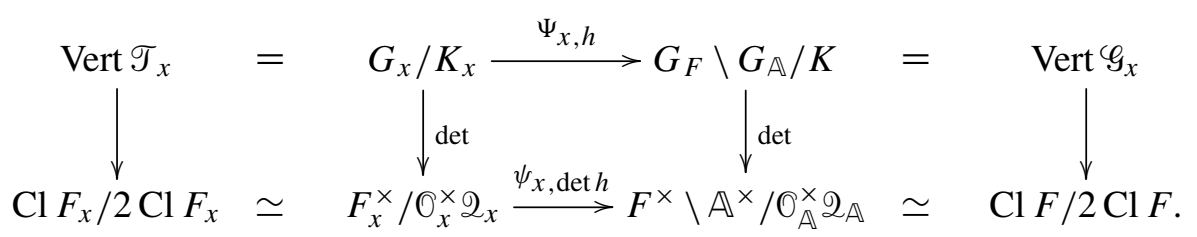

This means that vertices with equal labels map to vertices with equal labels.

Each component of $\mathscr{G}_{x}$ lies in the image of a suitable $\Psi_{x, h}$, and thus has at most two labels. On the other hand, the two labels of $\mathscr{T}_{x}$ map to $\psi_{x, \operatorname{det} h}([1])=[a]$ and $\psi_{x, \operatorname{det} h}\left(\left[\pi_{x}\right]\right)=\left[a \pi_{x}\right]$, where $a=\operatorname{det} h$. The divisor classes of $[a]$ and $\left[a \pi_{x}\right]$ differ by the class of the prime divisor $x$, and are equal if and only if $x$ is a square in the divisor class group. If so, according to Proposition 3.8, there must be $2 h_{2}$ components, so that the $2 h_{2}$ labels are spread over all components. If $x$ is not a square, then by the local surjectivity of $\Psi_{x, h}$ on edges two adjacent vertices of $\varphi_{x}$ also have different labels.

\section{Geometric interpretation of unramified Hecke operators}

A fundamental observation in the geometric Langlands program (for $\mathrm{PGL}_{2}$, in this case) is that the domain of automorphic forms (with a certain ramification level) corresponds to the isomorphism classes of $\mathbb{P}^{1}$-bundles (with a corresponding level structure). The action of Hecke operators can be given a geometric meaning, which makes it possible to let algebraic geometry enter the field. We will use this geometric view point for a closer examination of the graphs of unramified Hecke operators. We begin with recalling the geometric interpretation of unramified Hecke operators. For more reference, see [Gaitsgory 2003].

5.1. Let $O_{X}$ be the structure sheaf of the smooth projective curve $X$ and $\eta$ the generic point. We can identify the stalks $\mathscr{O}_{X, x}$ of the structure sheaf $O_{X}$ at closed points $x \in\|X\|$ and their embeddings into the generic stalk $\mathcal{O}_{X, \eta}$ with

$$
\mathcal{O}_{X, x} \simeq \mathcal{O}_{x} \cap F \hookrightarrow F \simeq \mathcal{O}_{X, \eta} .
$$

We identify vector bundles on $X$ with the corresponding locally free sheaf [Hartshorne 1977, Exercise II.5.18]. We denote by $\operatorname{Bun}_{n} X$ the set of isomorphism 
classes of rank $n$ bundles over $X$ and by Pic $X$ the Picard group. For $\mathscr{L}_{1}, \mathscr{L}_{2} \in \operatorname{Pic} X$, we use the shorthand notation $\mathscr{L}_{1} \mathscr{L}_{2}$ for $\mathscr{L}_{1} \otimes \mathscr{L}_{2}$. The group Pic $X$ acts on $\operatorname{Bun}_{n} X$ by tensor products. Let $\mathbb{P B u n}_{n} X$ be the orbit set $\operatorname{Bun}_{n} X / \operatorname{Pic} X$, which is nothing but the set of isomorphism classes of $\mathbb{P}^{n-1}$-bundles over $X$ [ibid., Ex. II.7.10].

We will call the elements of $\mathbb{P B u n}_{2} X$ projective line bundles. If we regard the total space of a projective line bundle as a scheme, then we obtain a ruled surface; see [ibid., Proposition V.2.2]. Thus $\mathbb{P B u n}_{2} X$ may also be seen as the set of isomorphism classes of ruled surfaces over $X$.

If two vector bundles $M_{1}$ and $M_{2}$ are in the same orbit of the action of Pic $X$, we write $M_{1} \sim M_{2}$ and say that $M_{1}$ and $M_{2}$ are projectively equivalent. When we say $[M] \in \mathbb{P B u n} 2 X$, we mean the class that is represented by the rank 2 bundle $\mathcal{M}$.

Let $\mathrm{Cl} X=\mathrm{Cl} F$ be the divisor group of $X$. Every divisor $D \in \mathrm{Cl} X$ defines the associated line bundle $\mathscr{L}_{D}$, which defines an isomorphism $\mathrm{Cl} X \rightarrow \operatorname{Pic} X$ of groups [ibid., Proposition II.6.15]. The degree $\operatorname{deg} M$ of a vector bundle $M$ with $\operatorname{det} \mathcal{M} \simeq \mathscr{L}_{D}$ is defined as $\operatorname{deg} D$; see [ibid., Ex. II.6.12]. For a torsion sheaf $\mathscr{F}$, the degree is defined by $\operatorname{deg} \mathscr{F}=\sum_{x \in\|X\|} \operatorname{dim}_{\mathbb{F}_{q}}\left(\mathscr{F}_{x}\right)$. The degree is additive in short exact sequences.

Remark 5.2. Note that if $D=x$ is a prime divisor, the notation for the associated line bundle $\mathscr{L}_{x}$ coincides with the notation for the stalk of $\mathscr{L}$ at $x$. In order to avoid confusion, we will reserve the notation $\mathscr{L}_{x}$ strictly for the associated line bundle. In case we have to consider the stalk of a line bundle, we will use a symbol different from $\mathscr{L}$ for the line bundle.

5.3. The correspondence between $\mathrm{Cl} X=F^{\times} \backslash \mathbb{A}^{\times} / \mathcal{O}_{\mathbb{A}}^{\times}$and Pic $X$ extends to higher rank. For more details on the following outline; see [Frenkel 2004, Lemma 3.1; Gaitsgory 2003, 2.1]. Let $\mathcal{M}$ be a rank 2 bundle. Then we can choose for every $x \in\|X\|$ a trivialization $\varphi_{x}$ of $\mu_{x}$ in a formal neighborhood of $x$, and a trivialization $\varphi_{\eta}$ of the generic stalk $\mu_{\eta}$. We define the matrix $g_{x}$ as the base change matrix corresponding to

$$
\mathcal{O}_{X, x}^{2} \stackrel{\varphi_{x}}{\longrightarrow} M_{x} \hookrightarrow M_{\eta} \stackrel{\varphi_{\eta}^{-1}}{\longrightarrow} F^{2}
$$

with respect to the standard bases of $\mathcal{O}_{X, x}^{2}$ and $F^{2}$. This yields an element $g=\left(g_{x}\right)$ of $\mathrm{GL}_{2}(\mathbb{A})$. A coordinate change of the stalks $M_{x}$ corresponds to a matrix in $\mathrm{GL}_{2}\left(\mathrm{O}_{\mathrm{A}}\right)$ and a coordinate change of $\mu_{\eta}$ corresponds to a matrix in $\mathrm{GL}_{2}(F)$. Indeed, every double coset in $\mathrm{GL}_{2}(F) \backslash \mathrm{GL}_{2}(\mathbb{A}) / \mathrm{GL}_{2}\left(\mathrm{O}_{\mathbb{A}}\right)$ is obtained from a vector bundle in the described way, which yields a bijection

$$
\begin{aligned}
\mathrm{GL}_{2}(F) \backslash \mathrm{GL}_{2}(\mathbb{A}) / \mathrm{GL}_{2}\left(\mathrm{O}_{\mathbb{A}}\right) & \stackrel{1: 1}{\longleftrightarrow} \operatorname{Bun}_{2} X, \\
{[g] } & \longmapsto M_{g}
\end{aligned}
$$


Furthermore, we have $M_{g} \otimes \mathscr{L}_{a}=\mathcal{M}_{a g}$ for $a \in \mathbb{A}^{\times}$, and $\operatorname{deg} M_{g}=\operatorname{deg}(\operatorname{det} g)$. Consequently, there is a bijection

$$
G_{F} \backslash G_{\mathrm{A}} / K \stackrel{1: 1}{\longleftrightarrow} \mathbb{P B u n}_{2} X,
$$

which allows us to identify the vertex set $\operatorname{Vert} \varphi_{x}=G_{F} \backslash G_{\mathrm{A}} / K$ with $\mathbb{P B u n} 2 X$.

5.4. The next task is to describe edges of $\mathscr{G}_{x}$ in geometric terms. We say that two exact sequences

$$
0 \rightarrow \mathscr{F}_{1} \rightarrow \mathscr{F}_{F} \rightarrow \mathscr{F}_{1}^{\prime} \rightarrow 0 \quad \text { and } \quad 0 \rightarrow \mathscr{F}_{2} \rightarrow \mathscr{F} \rightarrow \mathscr{F}_{2}^{\prime} \rightarrow 0
$$

of sheaves are isomorphic with fixed $\mathscr{F}_{\text {if }}$ if there are isomorphisms $\mathscr{F}_{1} \rightarrow \mathscr{F}_{2}$ and $\mathscr{F}_{1}^{\prime} \rightarrow \mathscr{F}_{2}^{\prime}$ such that

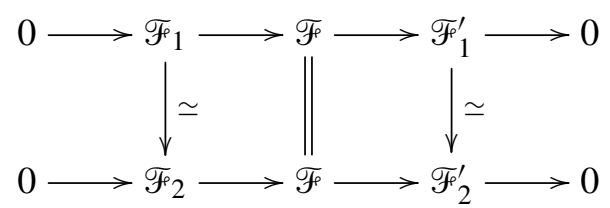

commutes.

Let $\mathscr{K}_{x}$ be the torsion sheaf that is supported at $x$ and has stalk $\kappa_{x}$ at $x$, where $\kappa_{x}$ is the residue field at $x$. Fix a representative $\mathcal{M}$ of $[M] \in \mathbb{P B u n} 2 X$. Then we define $m_{x}\left([\mathcal{M}],\left[\mathcal{M}^{\prime}\right]\right)$ as the number of isomorphism classes of exact sequences

$$
0 \rightarrow \mathcal{M}^{\prime \prime} \rightarrow M \rightarrow \mathscr{K}_{x} \rightarrow 0,
$$

with fixed $\mathcal{M}$ and with $\mathcal{M}^{\prime \prime} \sim \mathcal{M}^{\prime}$. This number is independent of the choice of the representative $\mathcal{M}$ because for another choice, which would be a vector bundle of the form $M \otimes \mathscr{L}$ for some $\mathscr{L} \in \operatorname{Pic} X$, we have the bijection

$$
\begin{aligned}
\left\{\begin{array}{c}
\text { isomorphism classes } \\
0 \rightarrow M^{\prime \prime} \rightarrow \mu \rightarrow \mathscr{K}_{x} \rightarrow 0 \\
\text { with fixed } \mathcal{M}
\end{array}\right\} & \rightarrow\left\{\begin{array}{c}
\text { isomorphism classes } \\
0 \rightarrow \mathcal{M}^{\prime \prime \prime} \rightarrow M \otimes \mathscr{L} \rightarrow \mathscr{K}_{x} \rightarrow 0 \\
\text { with fixed } M \otimes \mathscr{L}
\end{array}\right\}, \\
\left(0 \rightarrow \mathcal{M}^{\prime \prime} \rightarrow M \rightarrow \mathscr{K}_{x} \rightarrow 0\right) & \mapsto\left(0 \rightarrow \mathcal{M}^{\prime \prime} \otimes \mathscr{L} \rightarrow M \otimes \mathscr{L} \rightarrow \mathscr{K}_{x} \rightarrow 0\right) .
\end{aligned}
$$

Definition 5.5. Let $x$ be a place. For a projective line bundle $[M] \in \mathbb{P B u n} 2 X$ we define

$$
\mathcal{u}_{x}([\mathcal{M}])=\left\{\left([\mathcal{M}],\left[\mathcal{M}^{\prime}\right], m\right) \mid m=m_{x}\left([\mathcal{M}],\left[\mathcal{M}^{\prime}\right]\right) \neq 0\right\},
$$

and call the occurring $\left[\mathcal{M}^{\prime}\right]$ the $\Phi_{x}$-neighbors of $[\mathcal{M}]$, and $m_{x}\left([\mathcal{M}],\left[\mathcal{M}^{\prime}\right]\right)$ their multiplicity. 
5.6. We shall show that this concept of neighbors is the same as the one defined for classes in $G_{F} \backslash G_{\mathbb{A}} / K$ (Definition 1.4). Recall that in Proposition 2.3, we determined the $\Phi_{x}$-neighbors of a class $[g] \in G_{F} \backslash G_{\mathrm{A}} / K$ to be of the form $\left[g \xi_{w}\right]$ for a $w \in \mathbb{P}^{1}\left(\kappa_{x}\right)$. The elements $\xi_{w}$ define exact sequences

$$
0 \rightarrow \prod_{y \in\|X\|} \mathcal{O}_{X, y}^{2} \stackrel{\xi_{w}}{\rightarrow} \prod_{y \in\|X\|} \mathcal{O}_{X, y}^{2} \rightarrow \kappa_{x} \rightarrow 0
$$

of $\mathbb{F}_{q}$-modules and consequently an exact sequence $0 \rightarrow \mathcal{M}_{g \xi_{w}} \rightarrow \mathcal{M}_{g} \rightarrow \mathcal{K}_{x} \rightarrow 0$ of sheaves, where $M_{g \xi_{w}}$ and $M_{g}$ are the rank 2 bundles associated with $g \xi_{w}$ and $g$, respectively. This maps $w \in \mathbb{P}^{1}\left(\kappa_{x}\right)$ to the isomorphism class of $\left(0 \rightarrow M_{g \xi_{w}} \rightarrow\right.$ $\left.M_{g} \rightarrow \mathscr{K}_{x} \rightarrow 0\right)$ with fixed $\mathcal{M}_{g}$. On the other hand, as we have chosen a basis for the stalk at $x$, each isomorphism class of sequences $\left(0 \rightarrow \mathcal{M}^{\prime} \rightarrow \mathcal{M} \rightarrow \mathcal{K}_{x} \rightarrow 0\right)$ with fixed $\mathcal{M}$ defines an element in $\mathbb{P}\left(\mathbb{O}_{X, x}^{2} /\left(\pi_{x} \mathcal{O}_{X, x}\right)^{2}\right)=\mathbb{P}^{1}\left(\kappa_{x}\right)$, which gives back $w$.

Thus for every $x \in\|X\|$, the map

$$
u_{x}([g]) \rightarrow \mathcal{U}_{x}\left(\left[\mathcal{M}_{g}\right]\right), \quad\left([g],\left[g^{\prime}\right], m\right) \mapsto\left(\left[\mathcal{M}_{g}\right],\left[\mathcal{M}_{g^{\prime}}\right], m\right)
$$

is a well-defined bijection. We finally obtain the geometric description of the graph $\mathscr{G}_{x}$ of $\Phi_{x}$.

Proposition 5.7. Let $x \in\|X\|$. The graph $\mathscr{G}_{x}$ of $\Phi_{x}$ is described in geometric terms as

$$
\operatorname{Vert} \mathscr{G}_{x}=\mathbb{P B u n}_{2} X \quad \text { and } \quad \text { Edge } \mathscr{G}_{x}=\bigsqcup_{[\mathcal{M}] \in \mathbb{P B u n}_{2} X} u_{x}([\mu \mathcal{M}])
$$

Remark 5.8. This interpretation shows that the graphs that we consider are a global version of the graphs of Serre [2003, Chapter II.2]. We are looking at all rank 2 bundles on $X$ modulo the action of the Picard group of $X$ while Serre considers rank 2 bundles that trivialize outside a given place $x$ modulo line bundles that trivialize outside $x$. As already explained in Remark 3.6, we obtain a projection of the graph of Serre to the component of the trivial class $c_{0}$.

Serre describes his graphs as quotients of Bruhat-Tits trees by the action of the group $\Gamma=G_{\bigcirc_{F}^{x}}$ on both vertices and edges. This leads in general to multiple edges between vertices in the quotient graph; see for example [Serre 2003, 2.4.2c]. This does not happen with graphs of Hecke operators: There is at most one edge with given origin and terminus.

Relative to the action of $\Gamma$ on Serre's graphs, one can define the weight of an edge as the order of the stabilizer of its origin in the stabilizer of the edge. The projection from Serre's graphs to graphs of Hecke operators identifies all the different edges between two vertices, adding up their weights to obtain the weight of the image edge. 


\section{Description of vertices}

The aim of this section is to show that the set of isomorphism classes of projective line bundles over $X$ can be separated into subspaces corresponding to certain quotients of the divisor class group of $F$, the divisor class group of $\mathbb{F}_{q^{2}} F$ and geometrically indecomposable projective line bundles. We recall a series of facts about vector bundles.

6.1. A vector bundle $M$ is indecomposable if for every decomposition $M=M_{1} \oplus M_{2}$ into two subbundles $M_{1}$ and $M_{2}$, one factor is trivial and the other is isomorphic to $M$. The Krull-Schmidt theorem holds for the category of vector bundles over $X$, that is, every vector bundle $\mathcal{M}$ on $X$ defined over $\mathbb{F}_{q}$ has, up to permutation of factors, a unique decomposition into a direct sum of indecomposable subbundles; see [Atiyah 1956, Theorem 2].

The map $p: X^{\prime}=X \otimes \mathbb{F}_{q^{i}} \rightarrow X$ defines the inverse image or the constant extension of vector bundles

$$
p^{*}: \operatorname{Bun}_{n} X \rightarrow \operatorname{Bun}_{n} X^{\prime}, \quad M \mapsto p^{*} M .
$$

The isomorphism classes of rank $n$ bundles that after extension of constants to $\mathbb{F}_{q^{i}}$ become isomorphic to $p^{*} \mathcal{M}$ are classified by $H^{1}\left(\operatorname{Gal}\left(\mathbb{F}_{q^{i}} / \mathbb{F}_{q}\right), \operatorname{Aut}\left(\mu \otimes \mathbb{F}_{q^{i}}\right)\right)$; see [Arason et al. 1992, Section 1]. The algebraic group $\operatorname{Aut}\left(\mathcal{M} \otimes \mathbb{F}_{q^{i}}\right)$ is an open subvariety of the connected algebraic group $\operatorname{End}\left(M \otimes \mathbb{F}_{q^{i}}\right)$, and thus it is itself a connected algebraic group. As a consequence of Lang's theorem [1956, Corollary to Theorem 1], we have $H^{1}\left(\operatorname{Gal}\left(\mathbb{F}_{q^{i}} / \mathbb{F}_{q}\right), \operatorname{Aut}\left(M \otimes \mathbb{F}_{q^{i}}\right)\right)=1$.

Thus $p^{*}$ is injective. In particular, one can consider the constant extension to the geometric curve $\bar{X}=X \otimes \overline{\mathbb{F}}_{q}$ over an algebraic closure $\overline{\mathbb{F}}_{q}$ of $\mathbb{F}_{q}$. Then two vector bundles are isomorphic if and only if they are geometrically isomorphic, that is, if their constant extensions to $\bar{X}$ are isomorphic. We can therefore think of $\operatorname{Bun}_{n} X$ as a subset of $\operatorname{Bun}_{n} X^{\prime}$ and $\operatorname{Bun}_{n} \bar{X}$.

On the other hand, $p: X^{\prime} \rightarrow X$ defines the direct image or the trace of vector bundles

$$
p_{*}: \operatorname{Bun}_{n} X^{\prime} \rightarrow \operatorname{Bun}_{n i} X, \quad \mathcal{M} \mapsto p_{*} \mathcal{M} .
$$

We have $p_{*} p^{*} \mathcal{M} \simeq \mathcal{M}^{i}$ for $\mathcal{M} \in \operatorname{Bun}_{n} X$ and $p^{*} p_{*} \mathcal{M} \simeq \bigoplus \mathcal{M}^{\tau}$ for $\mathcal{M} \in \operatorname{Bun}_{n} X^{\prime}$, where $\tau$ ranges over $\operatorname{Gal}\left(\mathbb{F}_{q^{i}} / \mathbb{F}_{q}\right)$ and $\mathcal{M}^{\tau}$ is defined by the stalks $\mu_{x}^{\tau}=\mu_{\tau^{-1}(x)}$.

We call a vector bundle geometrically indecomposable if its extension to $\bar{X}$ is indecomposable. In [Arason et al. 1992, Theorem 1.8], it is shown that every indecomposable vector bundle over $X$ is the trace of a geometrically indecomposable bundle over some constant extension $X^{\prime}$ of $X$.

There are certain compatibilities of the constant extension and the trace with tensor products. Namely, for a vector bundle $\mathcal{M}$ and a line bundle $\mathscr{L}$ over $X$, we 
have $p^{*}(\mathcal{M} \otimes \mathscr{L}) \simeq p^{*} M \otimes p^{*} \mathscr{L}$ and for a vector bundle $\mathcal{M}^{\prime}$ over $X^{\prime}$,

$$
p_{*} \mathcal{M}^{\prime} \otimes \mathscr{L} \simeq p_{*}\left(\mathcal{M}^{\prime} \otimes p^{*} \mathscr{L}\right) .
$$

Thus $p^{*}$ induces a map, denoted by the same symbol,

$$
p^{*}: \mathbb{P B u n}_{n} X \rightarrow \mathbb{P B u n}_{n} X^{\prime}, \quad[\mathcal{M}] \mapsto\left[p^{*} \mathcal{M}\right],
$$

and $p_{*}$ induces

$$
p_{*}: \operatorname{Bun}_{n} X^{\prime} / p^{*} \operatorname{Pic} X \rightarrow \mathbb{P B u n}_{n i} X, \quad[\mathcal{M}] \mapsto\left[p_{*} \mathcal{M}\right] .
$$

6.2. We look at the situation for rank 2 bundles. Let $\sigma$ be the nontrivial automorphism of $\mathbb{F}_{q^{2}} / \mathbb{F}_{q}$. The set $\mathbb{P B u n}_{2} X$ is the disjoint union of the set of classes of decomposable rank 2 bundles, that is, rank 2 bundles that are isomorphic to the direct sum of two line bundles, and the set of classes of indecomposable bundles. We denote these sets by $\mathbb{P B u n}{ }_{2}^{\text {dec }} X$ and $\mathbb{P B u n}_{2}^{\text {indec }} X$, respectively. Let $\mathbb{P B u n}_{2}^{\mathrm{gi}} X \subset \mathbb{P B u n}_{2}^{\text {indec }} X$ be the subset of classes of geometrically indecomposable bundles. Since the rank is 2, the complement $\mathbb{P B u n}_{2}^{\text {tr }} X=\mathbb{P B u n}_{2}^{\text {indec }} X-\mathbb{P B u n}_{2}^{\text {gi }} X$ consists of classes of traces $p_{*} \mathscr{L}$ of certain line bundles $\mathscr{L} \in$ Pic $X^{\prime}$ that are defined over the quadratic extension $X^{\prime}=X \otimes \mathbb{F}_{q^{2}}$. More precisely, $p_{*} \mathscr{L}$ decomposes if and only if $\mathscr{L} \in p^{*}$ Pic $X$, and then $p_{*} \mathscr{L} \sim \mathscr{O}_{X} \oplus \mathscr{O}_{X}$. Thus, we have a disjoint union

$$
\mathbb{P B u n}_{2} X=\mathbb{P B u n}_{2}^{\mathrm{dec}} X \sqcup \mathbb{P B u n}{ }_{2}^{\mathrm{tr}} X \sqcup \mathbb{P B u n}{ }_{2}^{\mathrm{gi}} X .
$$

For $[D] \in \mathrm{Cl} X$, define

$$
c_{D}=\left[\mathscr{L}_{D} \oplus \mathscr{O}_{X}\right] \in \mathbb{P B u n}_{2}^{\mathrm{dec}} X,
$$

and for a $[D] \in \mathrm{Cl} X^{\prime}$, define

$$
t_{D}=\left[p_{*} \mathscr{L}_{D}\right] \in \mathbb{P B u n}{ }_{2}^{\text {tr }} X \cup\left\{c_{0}\right\} .
$$

Note that $\sigma$ acts on $\mathrm{Cl} X^{\prime}$ in a way compatible with the identification $\mathrm{Cl} X^{\prime} \simeq \operatorname{Pic} X^{\prime}$. Since $p^{*} p_{*}(\mathscr{L}) \simeq \mathscr{L} \oplus \mathscr{L}^{\sigma} \simeq p^{*} p_{*}\left(\mathscr{L}^{\sigma}\right)$ for $\mathscr{L} \in \operatorname{Pic} X^{\prime}$, and isomorphism classes of vector bundles are stable under constant extensions, we have $t_{D}=t_{\sigma D}$.

We derive the following characterizations of $\mathbb{P B u n}_{2}^{\text {dec }} X$ and $\mathbb{P B u n}{ }_{2}^{\text {tr }} X$ :

Proposition 6.3. The map $\mathrm{Cl} X \rightarrow \mathbb{P B u n}_{2}^{\mathrm{dec}} X,[D] \mapsto c_{D}$ is surjective with fibers of the form $\{[D],[-D]\}$.

Proof. Let $\mathcal{M}$ decompose into $\mathscr{L}_{1} \oplus \mathscr{L}_{2}$. Then

$$
\mu \simeq \mathscr{L}_{1} \oplus \mathscr{L}_{2} \sim\left(\mathscr{L}_{1} \oplus \mathscr{L}_{2}\right) \otimes \mathscr{L}_{2}^{-1} \simeq \mathscr{L}_{1} \mathscr{L}_{2}^{-1} \oplus \mathcal{O}_{X},
$$

thus surjectivity follows. Let $\mathscr{L}_{D^{\prime}} \oplus \mathcal{O}_{X}$ represent the same projective line bundle as $\mathscr{L}_{D} \oplus \mathcal{O}_{X}$. Then, there is a line bundle $\mathscr{L}_{0}$ such that $\mathscr{L}_{D} \oplus \mathcal{O}_{X} \simeq\left(\mathscr{L}_{D^{\prime}} \oplus \mathscr{O}_{X}\right) \otimes \mathscr{L}_{0}$, 
and thus either $\mathscr{L}_{0} \simeq \mathscr{O}_{X}$ and $\mathscr{L}_{D} \simeq \mathscr{L}_{D^{\prime}}$ or $\mathscr{L}_{0} \simeq \mathscr{L}_{D}$ and $\mathscr{L}_{D^{\prime}} \otimes \mathscr{L}_{D} \simeq \mathcal{O}_{X}$. Hence $\left[D^{\prime}\right]$ equals either $[D]$ or $[-D]$.

Proposition 6.4. The map $\mathrm{Cl} X^{\prime} / \mathrm{Cl} X \rightarrow \mathbb{P B u n}_{2}^{\mathrm{tr}} X \cup\left\{c_{0}\right\},[D] \mapsto t_{D}$ is surjective with fibers of the form $\{[D],[-D]\}$.

Proof. From the previous considerations it is clear that this map is well-defined and surjective. Assume that $\left[D_{1}\right],\left[D_{2}\right] \in \mathrm{Cl} X^{\prime}$ have the same image. Then there is an $\mathscr{L}_{0} \in \operatorname{Pic} X$ such that $p_{*} \mathscr{L}_{1} \simeq p_{*} \mathscr{L}_{2} \otimes \mathscr{L}_{0}$, where we briefly wrote $\mathscr{L}_{i}$ for $\mathscr{L}_{D_{i}}$. Then in $\mathbb{P B u n} 2 X^{\prime}$, we see that

$$
\mathscr{L}_{1} \oplus \mathscr{L}_{1}^{\sigma} \simeq p^{*} p_{*} \mathscr{L}_{1} \simeq p^{*} p_{*} \mathscr{L}_{2} \otimes p^{*} \mathscr{L}_{0} \simeq\left(\mathscr{L}_{2} \otimes p^{*} \mathscr{L}_{0}\right) \oplus\left(\mathscr{L}_{2}^{\sigma} \otimes p^{*} \mathscr{L}_{0}\right),
$$

thus either $\mathscr{L}_{1} \simeq \mathscr{L}_{2} \otimes p^{*} \mathscr{L}_{0}$, which implies that $D_{1}$ and $D_{2}$ represent the same class in $\mathrm{Cl} X^{\prime} / \mathrm{Cl} X$, or $\mathscr{L}_{1} \simeq \mathscr{L}_{2}^{\sigma} \otimes p^{*} \mathscr{L}_{0}$, which means that $D_{1}$ represents the same class as $\sigma D_{2}$. But in $\mathrm{Cl} X^{\prime} / \mathrm{Cl} X$,

$$
\left[\sigma D_{2}\right]=[\underbrace{\sigma D_{2}+D_{2}}_{\in \mathrm{Cl} X}-D_{2}]=\left[-D_{2}\right] .
$$

Lemma 6.5. The constant extension restricts to an injective map

$$
p^{*}: \mathbb{P B u n}_{2}^{\mathrm{dec}} X \sqcup \mathbb{P B u n}{ }_{2}^{\mathrm{tr}} X \hookrightarrow \mathbb{P B u n}_{2}^{\mathrm{dec}} X^{\prime} .
$$

Proof. Since $p^{*} p_{*}(\mathscr{L}) \simeq \mathscr{L} \oplus \mathscr{L}^{\sigma}$ for a line bundle $\mathscr{L}$ over $X^{\prime}$, it is clear that the image is contained in $\mathbb{P B u n}_{2}^{\mathrm{dec}} X^{\prime}$. The images of $\mathbb{P B u n}_{2}^{\mathrm{dec}} X$ and $\mathbb{P B u n}_{2}^{\mathrm{tr}} X$ are disjoint since elements of the image of the latter set decompose into line bundles over $X^{\prime}$ that are not defined over $X$. If we denote taking the inverse elements by inv, then by Proposition $6.3, p^{*}$ is injective restricted to $\mathbb{P B u n}_{2}^{\mathrm{dec}} X$ because $(\mathrm{Cl} X /$ inv $) \rightarrow\left(\mathrm{Cl} X^{\prime} /\right.$ inv $)$ is. Regarding $\mathbb{P B u n}_{2}^{\text {tr }} X$, observe that

$$
p^{*}\left(t_{D}\right)=p^{*} p_{*}\left(\mathscr{L}_{D}\right) \simeq \mathscr{L}_{D} \oplus \mathscr{L}_{\sigma D} \sim \mathscr{L}_{D-\sigma D} \oplus \mathcal{O}_{X^{\prime}}=c_{D-\sigma D},
$$

where by Proposition 6.4, D represents an element in $\left(\mathrm{Cl} X^{\prime} / \mathrm{Cl} X\right) /$ inv, and by

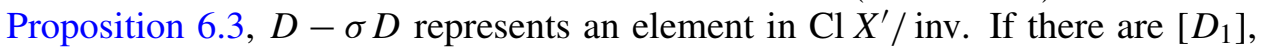
$\left[D_{2}\right] \in \mathrm{Cl} X^{\prime}$ such that $\left(D_{1}-\sigma D_{1}\right)= \pm\left(D_{2}-\sigma D_{2}\right)$, then $D_{1} \mp D_{2}=\sigma\left(D_{1} \mp D_{2}\right)$, and consequently $\left[D_{1} \mp D_{2}\right] \in \mathrm{Cl} X$.

Remark 6.6. The constant extension also restricts to a map

$$
p^{*}: \mathbb{P B u n}_{2}^{\mathrm{gi}} X \rightarrow \mathbb{P B u n}_{2}^{\mathrm{gi}} X^{\prime} .
$$

But this restriction is in general not injective in contrast to the previous result. For a counterexample to injectivity, see [Lorscheid 2012, Remark 2.7]. 


\section{Reduction theory for rank 2 bundles}

In this section, we introduce reduction theory for rank 2 bundles, that is, an invariant $\delta$ closely related to the slope of a vector bundle and reduction theory. Namely, a rank 2 bundle $\mathcal{M}$ is (semi)stable if and only if $\delta(M)$ is negative (nonpositive). For the definition of the slope of a vector bundle and (semi)stable vector bundles, see [Harder and Narasimhan 1974/75]. The invariant $\delta$ is also defined for projective line bundles and will be help to determine the structure of the graphs $\varphi_{x}$.

7.1. In general, the cokernel of a sheaf morphism between two vector bundles might have nontrivial torsion. A subbundle of a vector bundle $\mathcal{M}$ is an injective morphism $\mathcal{M}^{\prime} \rightarrow M$ of vector bundles such that the cokernel is again a vector bundle. By a line subbundle $\mathscr{L} \rightarrow M$ of a vector bundle $\mathcal{M}$, we mean a subbundle of $\mathcal{M}$ where $\mathscr{L}$ is a line bundle.

Every locally free subsheaf $\mathscr{L} \rightarrow \mathcal{M}$ of rank 1 extends to a uniquely determined line subbundle $\overline{\mathscr{L}} \rightarrow M$, since $\overline{\mathscr{L}}$ is determined by the constraint $\mathscr{L} \subset \overline{\mathscr{L}}$ [Serre 2003, p. 100]. On the other hand, every rank 2 bundle has a line subbundle [Hartshorne 1977, Corollary V.2.7].

Two line subbundles $\mathscr{L} \rightarrow \mathcal{M}$ and $\mathscr{L}^{\prime} \rightarrow \mathcal{M}$ are said to be the same if their images coincide, or, in other words, if there is an isomorphism $\mathscr{L} \simeq \mathscr{L}^{\prime}$ that commutes with the inclusions into $M$.

For a line subbundle $\mathscr{L} \rightarrow \mathcal{M}$ of a rank 2 bundle $\mathcal{M}$, we define

$$
\begin{aligned}
\delta(\mathscr{L}, \mathcal{M}) & :=\operatorname{deg} \mathscr{L}-\operatorname{deg}(\mathcal{M} / \mathscr{L})=2 \operatorname{deg} \mathscr{L}-\operatorname{deg} \mathcal{M}, \\
\delta(\mathcal{M}) & :=\sup _{\substack{\mathscr{L} \rightarrow \mathcal{M} \\
\text { line subbundle }}} \delta(\mathscr{L}, \mathcal{M}) .
\end{aligned}
$$

If $\delta(\mathcal{M})=\delta(\mathscr{L}, \mathcal{M})$, then we call $\mathscr{L}$ a line subbundle of maximal degree, or briefly, a maximal subbundle. Since $\delta\left(\mathscr{L} \otimes \mathscr{L}^{\prime}, M \otimes \mathscr{L}^{\prime}\right)=\delta(\mathscr{L}, M)$ for a line bundle $\mathscr{L}^{\prime}$, the invariant $\delta$ is well-defined on $\mathbb{P B u n}_{2} X$, and we put $\delta([\mathcal{M}])=\delta(\mathcal{M})$.

Let $g_{X}$ be the genus of $X$. Then the Riemann-Roch theorem and Serre duality imply:

Proposition 7.2 [Serre 2003, II.2.2, Propositions 6 and 7]. Every rank 2 bundle $M$ satisfies $-2 g_{X} \leq \delta(\mathcal{M})<\infty$. If $\mathscr{L} \rightarrow M$ is a line subbundle with $\delta(\mathscr{L}, \mathcal{M})>2 g_{X}-2$, then $\mathcal{M} \simeq \mathscr{L} \oplus M / \mathscr{L}$.

7.3. Every extension of a line bundle $\mathscr{L}^{\prime}$ by a line bundle $\mathscr{L}$, that is, every exact sequence of the form $0 \rightarrow \mathscr{L} \rightarrow M \rightarrow \mathscr{L}^{\prime} \rightarrow 0$, determines a rank 2 bundle $M \in \mathrm{Bun}_{2} X$. This defines for all $\mathscr{L}, \mathscr{L}^{\prime} \in \operatorname{Pic} X$ a map $\operatorname{Ext}^{1}\left(\mathscr{L}^{\prime}, \mathscr{L}\right) \rightarrow \operatorname{Bun}_{2} X$, which maps the zero element to $\mathscr{L} \oplus \mathscr{L}^{\prime}$. Since decomposable bundles may have line subbundles that differ from its given two factors, nontrivial elements can give rise to decomposable bundles. 
Proposition 7.4. The map

$$
\bigsqcup_{\substack{-2 g_{X} \leq \operatorname{deg} \mathscr{L} \\ \leq 2 g_{X}-2}} \operatorname{Ext}^{1}\left(\mathcal{O}_{X}, \mathscr{L}\right) \rightarrow \mathbb{P B u n} 2 X
$$

meets every element of $\mathbb{P B u n}{ }_{2}^{\text {indec }} X$, and the fiber of any $[M] \in \mathbb{P B u n} 2 X$ is of the form

$$
\left\{0 \rightarrow \mathscr{L} \rightarrow M \rightarrow O_{X} \rightarrow 0 \mid \delta(\mathscr{L}, M) \geq-2 g_{X}\right\} .
$$

Proof. We know that every $[\mathcal{M}] \in \mathbb{P B u n} 2 X$ has a reduction $0 \rightarrow \mathscr{L} \rightarrow \mathcal{M} \rightarrow \mathscr{L}^{\prime} \rightarrow 0$ with $\delta(\mathscr{L}, M) \geq-2 g_{X}$, where we may assume that $\mathscr{L}^{\prime}=0_{X}$ by replacing $M$ with $M \otimes\left(\mathscr{L}^{\prime}\right)^{-1}$; hence $\delta(\mathscr{L}, M)=\operatorname{deg} \mathscr{L}$. If $\operatorname{deg} \mathscr{L}>2 g_{X}-2$, then $M$ decomposes, that is, $\operatorname{Ext}^{1}\left(\mathrm{O}_{X}, \mathscr{L}\right)$ is trivial (which is already clear from the proof [Serre 2003, II.2.2, Proposition 7]). This explains the form of the fibers and that $\mathbb{P B u n}_{2}^{\text {indec }} X$ is contained in the image.

Corollary 7.5. There are only finitely many isomorphism classes of indecomposable projective line bundles.

Proof. This is clear since $\bigsqcup_{-2 g_{X} \leq \operatorname{deg} \mathscr{L} \leq 2 g_{X}-2} \operatorname{Ext}^{1}\left(O_{X}, \mathscr{L}\right)$ is a finite union of finite sets.

Lemma 7.6. If $\mathscr{L} \rightarrow \mathcal{M}$ is a maximal subbundle, then $\delta\left(\mathscr{L}^{\prime}, \mathcal{M}\right) \leq-\delta(\mathscr{L}, \mathcal{M})$ for every line subbundle $\mathscr{L}^{\prime} \rightarrow \mathcal{M}$ that is different from $\mathscr{L} \rightarrow M$. Equality holds if and only if $\mathcal{M} \simeq \mathscr{L} \oplus \mathscr{L}^{\prime}$, that is, $M$ decomposes and $\mathscr{L}^{\prime}$ is a complement of $\mathscr{L}$ in $M$.

Proof. Compare with [Schleich 1974, Lemma 3.1.1.]. Since $\mathscr{L}^{\prime} \rightarrow \mathcal{M}$ is different from $\mathscr{L} \rightarrow M$, there is no inclusion $\mathscr{L}^{\prime} \rightarrow \mathscr{L}$ that commutes with the inclusions into $M$. Hence the composed morphism $\mathscr{L}^{\prime} \rightarrow M \rightarrow M / \mathscr{L}$ must be injective, and $\operatorname{deg} \mathscr{L}^{\prime} \leq \operatorname{deg} \mathcal{M} / \mathscr{L}=\operatorname{deg} M-\operatorname{deg} \mathscr{L}$. This implies that

$$
\delta\left(\mathscr{L}^{\prime}, M\right)=2 \operatorname{deg} \mathscr{L}^{\prime}-\operatorname{deg} M \leq \operatorname{deg} M-2 \operatorname{deg} \mathscr{L}=-\delta(\mathscr{L}, M) .
$$

Equality holds if and only if $\mathscr{L}^{\prime} \rightarrow M / \mathscr{L}$ is an isomorphism, and in this case, its inverse defines a section $M / \mathscr{L} \simeq \mathscr{L}^{\prime} \rightarrow M$.

\section{Proposition 7.7.}

(i) A rank 2 bundle $M$ has at most one line subbundle $\mathscr{L} \rightarrow M$ such that $\delta(\mathscr{L}, M) \geq 1$.

(ii) If $\mathscr{L} \rightarrow \mathcal{M}$ is a line subbundle with $\delta(\mathscr{L}, \mathcal{M}) \geq 0$, then $\delta(\mathcal{M})=\delta(\mathscr{L}, \mathcal{M})$.

(iii) If $\delta(M)=0$, we distinguish three cases.

(1) $\mathcal{M}$ has only one maximal line bundle; this happens if and only if $\mathcal{M}$ is indecomposable.

(2) $\mathcal{M}$ has exactly two maximal subbundles $\mathscr{L}_{1} \rightarrow \mathcal{M}$ and $\mathscr{L}_{2} \rightarrow \mathcal{M}$; this happens if and only if $\mathcal{M} \simeq \mathscr{L}_{1} \oplus \mathscr{L}_{2}$ and $\operatorname{deg} \mathscr{L}_{1}=\operatorname{deg} \mathscr{L}_{2}$, but $\mathscr{L}_{1} \nsucceq \mathscr{L}_{2}$. 
(3) $M$ has exactly $q+1$ maximal subbundles; this happens if and only if all maximal subbundles are of the same isomorphism type $\mathscr{L}$ and $\mathcal{M} \simeq \mathscr{L} \oplus \mathscr{L}$.

(iv) $\delta\left(c_{D}\right)=\|\operatorname{deg} D\|$.

(v) $\delta(\mathcal{M})$ is invariant under extension of constants for $[M] \in \mathbb{P B u n}{ }_{2}^{\mathrm{dec}} X$.

Proof. Everything follows from the preceding lemmas, except for the fact that $\mathscr{L} \oplus \mathscr{L}$ has precisely $q+1$ maximal subbundles in part (3), which needs some explanation.

If $M=\mathscr{L} \oplus \mathscr{L}$ and $\mathscr{L}^{\prime}$ is a third maximal subbundle of $M$, then $M \simeq \mathscr{L}^{\prime} \oplus \mathscr{L}$ by Lemma 7.6, and thus there is an automorphism $M \simeq \mathscr{L}^{\prime} \oplus \mathscr{L} \rightarrow \mathscr{L} \oplus \mathscr{L}=M$ that restricts to an isomorphism between $\mathscr{L}^{\prime}$ and $\mathscr{L}$ by the Krull-Schmidt theorem; see [Atiyah 1956]. Thus the automorphism group $\operatorname{Aut}(\mathcal{M})$ of $\mathcal{M}$ acts transitively on the set of maximal line bundles of $\mathcal{M}$. Since $\operatorname{Aut}(\mathcal{M}) \simeq \mathrm{GL}_{2}\left(\mathbb{F}_{q}\right)$, the orbit of a maximal subbundle under $\operatorname{Aut}(\mathcal{M})$ is of cardinality $q+1$.

Proposition 7.8. Let $p: X^{\prime}=X \otimes \mathbb{F}_{q^{2}} \rightarrow X$ and $\mathscr{L} \in \operatorname{Pic} X^{\prime}$, then $\delta\left(p_{*} \mathscr{L}\right)$ is an even nonpositive integer. It equals 0 if and only if $\mathscr{L} \in p^{*} \mathrm{Pic} X$.

Proof. Over $X^{\prime}$, we have $p^{*} p_{*} \mathscr{L} \simeq \mathscr{L} \oplus \mathscr{L}^{\sigma}$ and $\operatorname{deg} \mathscr{L}=\operatorname{deg} \mathscr{L}^{\sigma}$. If $\mathscr{L}^{\prime}$ is a line subbundle of $p_{*} \mathscr{L}$, then $p^{*} \mathscr{L}^{\prime}$ is a subbundle of $\mathscr{L} \oplus \mathscr{L}^{\sigma}$. By the previous proposition, the degree of $p^{*} \mathscr{L}^{\prime}$ (which is the same as the degree of $\mathscr{L}^{\prime}$ ) equals the degree of $\mathscr{L}$ if and only if $p^{*} \mathscr{L}^{\prime}$ is isomorphic to $\mathscr{L}$ or $\mathscr{L}^{\sigma}$, and it is smaller otherwise. In the former case, $\mathscr{L}$ is already defined over $X$; thus $p_{*} \mathscr{L} \simeq \mathscr{L}^{\prime} \oplus \mathscr{L}^{\prime}$ and $\delta\left(p_{*} \mathscr{L}\right)=0$ if $\mathscr{L} \simeq p^{*} \mathscr{L}^{\prime}$. In the latter case, that is, if $\mathscr{L}$ is not of the form $p^{*} \mathscr{L}^{\prime}$ for a line bundle $\mathscr{L}^{\prime}$ over $X$, we have $\delta\left(\mathscr{L}^{\prime}, p_{*} \mathscr{L}\right)<0$ for every maximal subbundle $\mathscr{L}^{\prime}$ of $p_{*} \mathscr{L}$. This shows that $\delta\left(p_{*} \mathscr{L}\right)$ is nonpositive, and that it is 0 if and only if $\mathscr{L} \in p^{*} \operatorname{Pic} X$.

Finally note that by the very definition of $\delta(\mathcal{M})$ for rank 2 bundles $\mathcal{M}$, it follows that $\delta(\mathcal{M}) \equiv \operatorname{deg} \mathcal{M}(\bmod 2)$, and $\operatorname{deg}\left(p_{*} \mathscr{L}\right)=2 \operatorname{deg} \mathscr{L}$ is even.

Remark 7.9. We see that for $[M] \in \mathbb{P B u n}{ }_{2}^{\text {tr }} X$, the invariant $\delta(\mathcal{M})$ must get larger if we extend constants to $\mathbb{F}_{q^{2}}$, because $p^{*}(\mathcal{M})$ decomposes over $X^{\prime}$. This stays in contrast to the result for classes in $\mathbb{P B u n}_{2}^{\mathrm{dec}} X$ (Proposition 7.7 (v)).

\section{Nucleus and cusps}

In this section, we will define certain subgraphs of $\varphi_{x}$ for a place $x$, namely, the cusp of a divisor class modulo $x$, which is an infinite subgraph of a simple nature, and the nucleus, which is a finite subgraph that depends heavily on the arithmetic of $F$. Finally, $\mathscr{G}_{x}$ can be described as the union of the nucleus with a finite number of cusps.

8.1. We use reduction theory to investigate sequences of the form

$$
0 \rightarrow \mathcal{M}^{\prime} \rightarrow \mathcal{M} \rightarrow \mathcal{K}_{x} \rightarrow 0,
$$


which occur in the definition of $\mho_{x}([\mathcal{M}])$. By additivity of the degree map (see Section 5.1), $\operatorname{deg} \mathcal{M}^{\prime}=\operatorname{deg} \mathcal{M}-d_{x}$ where $d_{x}$ is the degree of $x$.

Given an arbitrary inclusion $\mathcal{M}^{\prime} \rightarrow \mathcal{M}$ of rank 2 bundles and a line subbundle $\mathscr{L} \rightarrow \mathcal{M}$, then we say that $\mathscr{L}$ lifts to $\mathcal{M}^{\prime}$ if there exists a morphism $\mathscr{L} \rightarrow \mathcal{M}^{\prime}$ such that the diagram

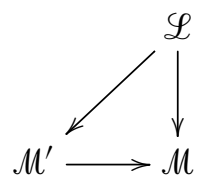

commutes. In this case, $\mathscr{L} \rightarrow \mathcal{M}^{\prime}$ is indeed a subbundle since otherwise it would extend nontrivially to a subbundle $\overline{\mathscr{L}} \rightarrow \mathcal{M}^{\prime} \subset \mathcal{M}$ and would contradict the hypothesis that $\mathscr{L}$ is a subbundle of $\mathcal{M}$. In the case that $\mathcal{M}^{\prime} \rightarrow \mathcal{M}$ is part of an exact sequence

$$
0 \rightarrow \mathcal{M}^{\prime} \rightarrow M \rightarrow \mathscr{K}_{x} \rightarrow 0,
$$

a line subbundle $\mathscr{L} \rightarrow M$ lifts to $\mathcal{M}^{\prime}$ if and only if the image of $\mathscr{L}$ in $\mathscr{K}_{x}$ is 0 .

Let $\mathscr{I}_{x} \subset \mathcal{O}_{X}$ be the kernel of $\mathcal{O}_{X} \rightarrow \mathscr{K}_{x}$. This is also a line bundle, since $\mathscr{K}_{x}$ is a torsion sheaf. For every line bundle $\mathscr{L}$, we may think of $\mathscr{L} \mathscr{I}_{x}$ as a subsheaf of $\mathscr{L}$. In Pic $X$, the line bundle $\mathscr{I}_{x}$ represents the inverse of $\mathscr{L}_{x}$, the line bundle associated with the divisor $x$. In particular, $\operatorname{deg} \Phi_{x}=\operatorname{deg} \mathscr{L}_{x}^{-1}=-d_{x}$.

If $\mathscr{L} \rightarrow M$ does not lift to a subbundle of $\mathcal{M}^{\prime}$, we have that $\mathscr{L} \Phi_{x} \subset \mathscr{L} \rightarrow M$ lifts to a subbundle of $\mathcal{M}^{\prime}$ :

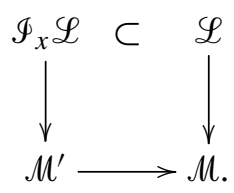

Note that every subbundle $\mathscr{L} \rightarrow \mathcal{M}^{\prime}$ is a locally free subsheaf $\mathscr{L} \rightarrow M$, which extends to a subbundle $\overline{\mathscr{L}} \rightarrow \mathcal{M}$. If thus $\mathscr{L} \rightarrow \mathcal{M}$ is a maximal subbundle that lifts to a subbundle $\mathscr{L} \rightarrow M^{\prime}$, then $\mathscr{L} \rightarrow \mathcal{M}^{\prime}$ is a maximal subbundle. If, however, $\mathscr{L} \rightarrow M$ is a maximal subbundle that does not lift to a subbundle $\mathscr{L} \rightarrow \mathcal{M}^{\prime}$, then $\mathscr{L} \mathscr{I}_{x} \rightarrow \mathcal{M}^{\prime}$ is a subbundle, which is not necessarily maximal. These considerations imply that

$$
\begin{aligned}
& \delta\left(\mathcal{M}^{\prime}\right) \leq 2 \operatorname{deg} \mathscr{L}-\operatorname{deg} \mathcal{M}^{\prime}=2 \operatorname{deg} \mathscr{L}-\left(\operatorname{deg} \mathcal{M}-d_{x}\right)=\delta(\mathcal{M})+d_{x}, \\
& \delta\left(\mathcal{M}^{\prime}\right) \geq 2 \operatorname{deg} \Phi_{x} \mathscr{L}-\operatorname{deg} \mathcal{M}^{\prime}=2 \operatorname{deg} \mathscr{L}-2 d_{x}-\left(\operatorname{deg} \mathcal{M}-d_{x}\right)=\delta(\mathcal{M})-d_{x} .
\end{aligned}
$$

Since $\delta\left(\mathcal{M}^{\prime}\right) \equiv \operatorname{deg} \mathcal{M}^{\prime}=\operatorname{deg} \mathcal{M}-d_{x}(\bmod 2)$, we derive the following:

Lemma 8.2. If $0 \rightarrow \mathcal{M}^{\prime} \rightarrow \mathcal{M} \rightarrow \mathscr{K}_{x} \rightarrow 0$ is exact, then

$$
\delta\left(\mathcal{M}^{\prime}\right) \in\left\{\delta(\mathcal{M})-d_{x}, \delta(\mathcal{M})-d_{x}+2, \ldots, \delta(\mathcal{M})+d_{x}\right\} .
$$


8.3. Every line subbundle $\mathscr{L} \rightarrow \mathcal{M}$ defines a line $\mathscr{L} / \mathscr{L} \mathscr{I}_{x}$ in $\mathbb{P}\left(\mathcal{M} /\left(\mathcal{M} \otimes \Phi_{x}\right)\right)$. By the bijection

$$
\begin{gathered}
\left\{\begin{array}{c}
\text { isomorphism classes of exact } \\
0 \rightarrow \mathcal{M}^{\prime} \rightarrow \mathcal{M} \rightarrow \mathscr{K}_{x} \rightarrow 0 \\
\text { with fixed } \mathcal{M}
\end{array}\right\} \stackrel{1: 1}{\rightarrow} \mathbb{P}\left(\mathcal{M} /\left(\mathcal{M} \otimes \mathscr{I}_{x}\right)\right), \\
\left(0 \rightarrow \mathcal{M}^{\prime} \rightarrow \mathcal{M} \rightarrow \mathscr{K}_{x} \rightarrow 0\right) \mapsto \mathcal{M}^{\prime} /\left(\mathcal{M} \otimes \Phi_{x}\right)
\end{gathered}
$$

(see Section 5.6), there is a unique $0 \rightarrow \mathcal{M}^{\prime} \rightarrow \mathcal{M} \rightarrow \mathscr{K}_{x} \rightarrow 0$ up to isomorphism with fixed $\mathcal{M}$, such that $\mathcal{M}^{\prime} /\left(M \otimes \Phi_{x}\right)=\mathscr{L} / \mathscr{L} \mathscr{I}_{x}$ in $\mathbb{P}\left(\mathcal{M} /\left(\mathcal{M} \otimes \mathscr{I}_{x}\right)\right)$. This means that $\mathscr{L}$ is contained in the image of $\mathcal{M}^{\prime} \rightarrow \mathcal{M}$ and that $\mathscr{L} \rightarrow \mathcal{M}$ lifts to a line subbundle $\mathscr{L} \rightarrow \mathcal{M}^{\prime}$. We call $0 \rightarrow \mathcal{M}^{\prime} \rightarrow \mathcal{M} \rightarrow \mathcal{K}_{x} \rightarrow 0$ the sequence associated with $\mathscr{L} \rightarrow \mathcal{M}$ relative to $\Phi_{x}$, or for short the associated sequence, and [ $\left.\mathcal{M}^{\prime}\right]$ the associated $\Phi_{x}$-neighbor. It follows that $\delta\left(\mathcal{M}^{\prime}\right) \geq \delta(\mathscr{L}, \mathcal{M})+d_{x}$.

We summarize this.

Lemma 8.4. If $\mathscr{L} \rightarrow M$ is a maximal subbundle, then the associated $\Phi_{x}$-neighbor $\left[\mathcal{M}^{\prime}\right]$ has $\delta\left(\mathcal{M}^{\prime}\right)=\delta(\mathcal{M})+d_{x}$. Therefore,

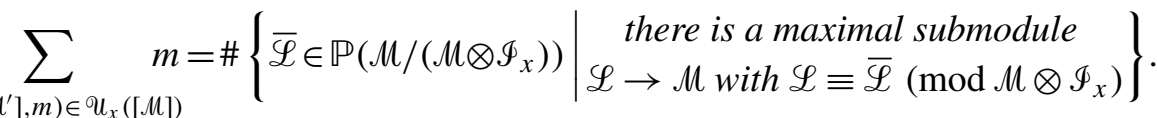

$$
\begin{aligned}
& \left([\mathcal{M}],\left[\mathcal{M}^{\prime}\right], m\right) \in \mathcal{U}_{x}([\mathcal{M}]) \\
& \delta\left(\mathcal{M}^{\prime}\right)=\delta(\mathcal{M})+d_{x}
\end{aligned}
$$

Theorem 8.5. Let $x$ be a place and $[D] \in \mathrm{Cl} X$ be a divisor of nonnegative degree. The $\Phi_{x}$-neighbors $v$ of $c_{D}$ with $\delta(v)=\operatorname{deg} D+d_{x}$ are given by the following list:

$$
\begin{aligned}
& \left(c_{0}, c_{x}, q+1\right) \in U_{x}\left(c_{0}\right), \\
& \left(c_{D}, c_{D+x}, 2\right) \in \mathcal{U}_{x}\left(c_{D}\right) \text { if }[D] \in\left(\mathrm{Cl}^{0} X\right)[2]-\{0\}, \\
& \left(c_{D}, c_{D+x}, 1\right),\left(c_{D}, c_{-D+x}, 1\right) \in \mathcal{U}_{x}\left(c_{D}\right) \quad \text { if }[D] \in \mathrm{Cl}^{0} X-\left(\mathrm{Cl}^{0} X\right)[2] \text {, } \\
& \left(c_{D}, c_{D+x}, 1\right) \in U_{x}\left(c_{D}\right) \text { if } \operatorname{deg} D \text { is positive. }
\end{aligned}
$$

For all $\Phi_{x}$-neighbors $v$ of $c_{D}$ not occurring in this list, $\delta(v)<\delta\left(c_{D}\right)+d_{x}$. If furthermore $\operatorname{deg} D>d_{x}$, then $\delta(v)=\operatorname{deg} D-d_{x}$, and if $\operatorname{deg} D>m_{X}+d_{x}$ where $m_{X}=\max \left\{2 g_{X}-2,0\right\}$, then

$$
U_{x}\left(c_{D}\right)=\left\{\left(c_{D}, c_{D-x}, q_{x}\right),\left(c_{D}, c_{D+x}, 1\right)\right\} .
$$

Proof. By Lemma 8.4, the $\Phi_{x}$-neighbors $v$ of $c_{D}$ with $\delta(v)=\delta\left(c_{D}\right)+d_{x}$ counted with multiplicity correspond to the maximal subbundles of a rank 2 bundle $M$ that represents $c_{D}$. Since $\delta(M)=\delta\left(c_{D}\right) \geq 0$, the list of all $\Phi_{x}$-neighbors $v$ of $c_{D}$ with $\delta(v)=\operatorname{deg} D+d_{x}=\delta\left(c_{D}\right)+d_{x}$ follows from the different cases in Proposition 7.7 (i) and (iii). Be aware that $c_{D}=c_{-D}$ by Proposition 6.3; hence it makes a difference whether or not $D$ is 2-torsion. 
For the latter statements, write $\mathcal{M}=\mathscr{L}_{D} \oplus \mathrm{O}_{X}$ and let $\mathcal{M}^{\prime}$ be a subsheaf of $\mathcal{M}$ with cokernel $\mathscr{K}_{x}$ such that $\delta\left(\mathcal{M}^{\prime}\right)<\delta(\mathcal{M})+d_{x}$. Then $\mathscr{L}_{D} \rightarrow \mathcal{M}$ does not lift to $\mathcal{M}^{\prime}$, but $\mathscr{L}_{D} \mathscr{I}_{x} \rightarrow \mathcal{M}^{\prime}$ is a line subbundle and

$$
\mathcal{M}^{\prime} / \mathscr{L}_{D} \mathscr{I}_{x} \simeq\left(\operatorname{det} \mathcal{M}^{\prime}\right)\left(\mathscr{L}_{D} \mathscr{I}_{x}\right)^{\vee} \simeq(\operatorname{det} \mathcal{M}) \mathscr{I}_{x}\left(\mathscr{L}_{D} \mathscr{I}_{x}\right)^{\vee} \simeq \mathscr{L}_{D} \mathscr{I}_{x}\left(\mathscr{L}_{D} \mathscr{I}_{x}\right)^{\vee} \simeq \mathfrak{O}_{X} .
$$

If $\operatorname{deg} D>d_{x}$, then

$$
\delta\left(\mathscr{L}_{D} \mathscr{\Im}_{x}, \mathcal{M}^{\prime}\right)=\operatorname{deg} \mathscr{L}_{D} \mathscr{I}_{x}-\operatorname{deg} \mathscr{O}_{X}=\operatorname{deg} D-d_{x}>0 .
$$

Proposition 7.7(i) implies that $\mathscr{L}_{D} \rightarrow \mathcal{M}$ is the unique maximal subbundle of $\mathcal{M}^{\prime}$ and thus $\delta\left(\mathcal{M}^{\prime}\right)=\delta(\mathcal{M})-d_{x}$.

If $\delta(\mathcal{M})>m_{X}+d_{x}$, then $\delta\left(\mathcal{M}^{\prime}\right)>m_{X} \geq 2 g_{X}-2$; hence $\mathcal{M}^{\prime}$ decomposes and represents $c_{D-x}$. Since the multiplicities of all $\Phi_{x}$-neighbors of a vertex sum up to $q_{x}+1$, this proves the last part of our assertions.

Definition 8.6. Let $x$ be a place. Let the divisor $D$ represent a class

$$
[D] \in \mathrm{ClO}_{X}^{x}=\mathrm{Cl} X /\langle x\rangle \text {. }
$$

We define the $\operatorname{cusp} \mathscr{C}_{x}(D)$ ( of $D$ in $\mathscr{G}_{x}$ ) as the full subgraph of $\mathscr{G}_{x}$ with vertices

$$
\operatorname{Vert} \mathscr{C}_{x}(D)=\left\{c_{D^{\prime}} \mid\left[D^{\prime}\right] \equiv[D](\bmod \langle x\rangle), \text { and } \operatorname{deg} D^{\prime}>m_{X}\right\},
$$

and the nucleus $\mathcal{N}_{x}\left(\right.$ of $\left.\varphi_{x}\right)$ as the full subgraph of $\mathscr{G}_{x}$ with vertices

$$
\operatorname{Vert} \mathcal{N}_{x}=\left\{[\mathcal{M}] \in \operatorname{PBun}_{2} X \mid \delta(\mathcal{M}) \leq m_{X}+d_{x}\right\} .
$$

8.7. Theorem 8.5 determines all edges of a cusp $\mathscr{C}_{x}(D)$. If $m_{X}<\operatorname{deg} D \leq m_{X}+d_{x}$, the cusp can be illustrated as below. Note that a cusp is an infinite graph. It has a regular pattern that repeats periodically. In diagrams we draw the pattern and indicate its periodic continuation with dots.

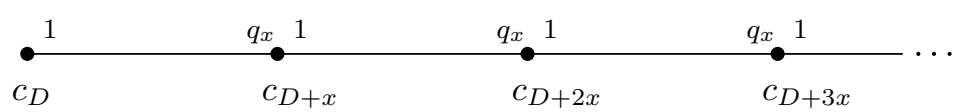

We summarize the theory so far in the following theorem that describes the general structure of $\varphi_{x}$.

Theorem 8.8. Let $x$ be a place of degree $d_{x}$ and $h_{X}=\# \mathrm{Cl}^{0} X$ be the class number.

(i) $\mathscr{G}_{x}$ has $h_{X} d_{x}$ cusps and

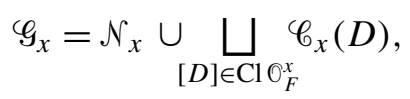

where Vert $\mathcal{N}_{x} \cap \operatorname{Vert} \mathscr{C}_{x}(D)=\left\{c_{D}\right\}$ if $m_{X}<\operatorname{deg} D \leq m_{X}+d_{x}$. The union of the edges is disjoint. Different cusps are disjoint subgraphs. 
(ii) $\mathcal{N}_{x}$ is finite and has $\#\left(\mathrm{ClO}_{F}^{x} / 2 \mathrm{ClO}_{F}^{x}\right)$ components. Each vertex of $\mathcal{N}_{x}$ is at distance $\leq\left(2 g_{X}+m_{X}+d_{x}\right) / d_{x}$ from some cusp. The associated $C W$-complexes of $\mathcal{N}_{x}$ and $\mathscr{G}_{x}$ are homotopy equivalent.

(iii) If $[D] \in \mathrm{ClO}_{F}^{x}$, then Vert $\mathscr{C}_{x}(D) \subset \mathbb{P B u n}{ }_{2}^{\text {dec }} X$. Furthermore,

$$
\begin{aligned}
\operatorname{PBun}_{2}^{\mathrm{dec}} X \subset\left\{v \in \operatorname{Vert} \mathscr{G}_{x} \mid \delta(v) \geq 0\right\}, \\
\operatorname{PBun}_{2}^{\mathrm{gi}} X \subset\left\{v \in \operatorname{Vert} \mathscr{G}_{x} \mid \delta(v) \leq 2 g_{X}-2\right\}, \\
\mathbb{P B u n}_{2}^{\text {tr }} X \subset\left\{v \in \operatorname{Vert} \mathscr{G}_{x} \mid \delta(v)<0 \text { and even }\right\} .
\end{aligned}
$$

8.9. Remark on Figure 8a. Define $h=h_{X}, m=m_{X}, d=d_{x}$ and $q_{x}=q^{\operatorname{deg} x}$. Further let $D_{1}, \ldots, D_{h d}$ be representatives for $\mathrm{ClO}_{F}^{x}$ with $m<\operatorname{deg} D_{i} \leq m+d$ for $i=1, \ldots, h d$. The cusps $\mathscr{C}_{x}\left(D_{i}\right)$ for $i=1, \ldots, h d$ can be seen in Figure $8 \mathrm{a}$ as the subgraphs in the dashed regions that are open to the right. The nucleus $\mathcal{N}_{x}$ is contained in the dashed rectangle to the left. Since we have no further information about the nucleus, we leave the area in the rectangle open.

The $\delta$-line on the bottom of the picture indicates the value $\delta(v)$ for the vertices $v$ in the graph that lie vertically above $\delta(v)$.

The dotted regions refer to the sort of vertices, which are elements of either $\mathbb{P B u n}_{2}^{\mathrm{gi}} X, \mathbb{P B u n}_{2}^{\mathrm{tr}} X$, or $\mathbb{P B u n}_{2}^{\mathrm{dec}} X$. All lines are drawn with reference to the $\delta$-line to reflect part (iii) of the theorem.

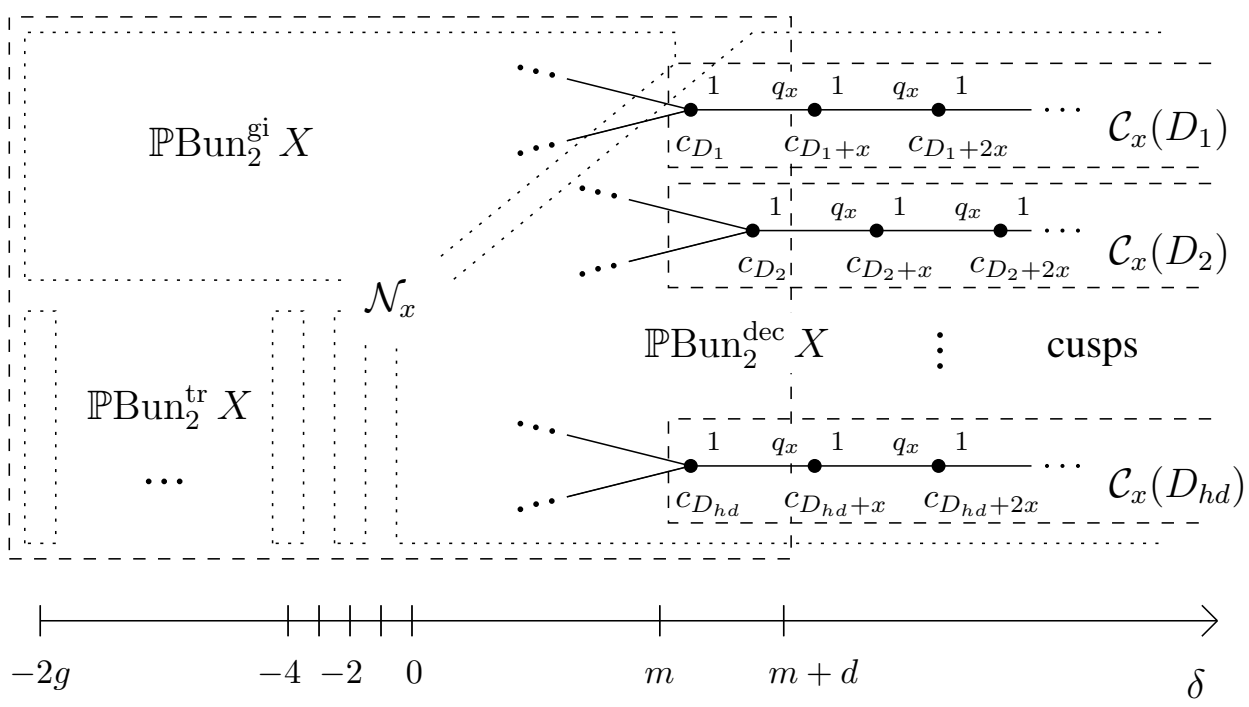

Figure 8a. General structure of $\mathscr{G}_{x}$. 
Proof. The number of cusps is $\# \mathrm{ClO}_{X}^{x}=\#(\mathrm{Cl} X /\langle x\rangle)=\# \mathrm{Cl}^{0} X \cdot \#\left(\mathbb{Z} / d_{x} \mathbb{Z}\right)=h_{X} d_{x}$. That the vertices of cusps are disjoint and only intersect in the given point with the nucleus is clear by definition. Regarding the edges, recall from Section 3.2 that if there is an edge from $v$ to $w$ in $\mathscr{G}_{x}$, then there is also an edge from $w$ to $v$. But Theorem 8.5 implies that each vertex of a cusp that does not lie in the nucleus only connects to a vertex of the same cusp; hence every edge of $\mathscr{G}_{x}$ either lies in a cusp or in the nucleus. Different cusps are disjoint by definition. This shows (i).

The nucleus is finite since $\mathbb{P B u n}_{2}^{\text {indec }} X$ is finite by Corollary 7.5 and since the intersection $\mathbb{P B u n}_{2}^{\operatorname{dec}} X \cap \operatorname{Vert} \mathcal{N}_{x}$ is finite by the definition of the nucleus and Proposition 6.3. Since the cusps are contractible as CW-complexes, $\mathcal{N}_{x}$ and $\mathscr{G}_{x}$ have the same homotopy type. Therefore $\mathcal{N}_{x}$ has $\#\left(\mathrm{ClO}_{F}^{x} / 20_{F}^{x}\right)$ components by Proposition 3.8. By Lemma 8.4, every vertex $v$ has a $\Phi_{x}$-neighbor $w$ with $\delta(w)=\delta(v)+d_{x}$, which is the upper bound for the distance of vertices in the nucleus to one of the cusps. This proves (ii).

The four statements of (iii) follow from the definition of a cusp, Propositions 7.7(iv), 7.2 and 7.8, respectively.

Example 8.10 (the projective line). Let $X$ be the projective line over $\mathbb{F}_{q}$. Then $g_{X}=0, h_{X}=1$ and $X$ has a closed point $x$ of degree 1 . This means that

$$
\operatorname{PBun}_{2}^{\mathrm{dec}} X=\left\{c_{n x}\right\}_{n \geq 0} .
$$

Since an indecomposable bundle $M$ must satisfy both $\delta(M) \geq 0$ and $\delta(\mathcal{M}) \leq-2$, which is impossible, all projective line bundles decompose. Theorem 8.5 together with the fact that the weights around each vertex sum to $q+1$ in the graph of $\Phi_{x}$ determines $\mathscr{G}_{x}$ completely, as illustrated here:

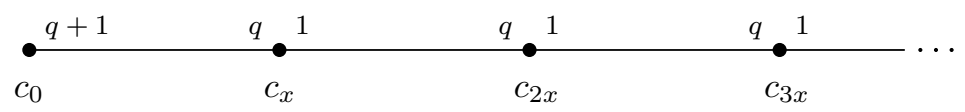

\section{Application to automorphic forms}

In this section, we explain how to recover automorphic forms as functions on the graph and indicate how unramified automorphic forms can be explicitly calculated as functions on the graph by solving a finite system of linear equations. We begin by recalling the definition of an automorphic form.

9.1. A function $f \in C^{0}\left(G_{\mathrm{A}}\right)$ is called an automorphic form (for $\mathrm{PGL}_{2}$ over $F$ ) if there is a compact open subgroup $K^{\prime}$ of $G_{\AA}$ such that $f$ is left $G_{F}$-invariant and right $K^{\prime}$-invariant and if it generates a finite-dimensional $\mathscr{H}_{K^{\prime}}$-subrepresentation $\mathscr{H}_{K^{\prime}}(f)$ of $C^{0}\left(G_{A}\right)$. We denote the space of automorphic forms by $\mathscr{A}$ and note that the action of $\mathscr{H}$ on $C^{0}\left(G_{\mathbb{A}}\right)$ restricts to $\mathscr{A}$. We denote the subspace of right $K^{\prime}$-invariant automorphic forms by $\mathscr{A}^{K^{\prime}}$, a space on which $\mathscr{H}_{K^{\prime}}$ acts. We can reinterpret the 
elements in $\mathscr{A}^{K^{\prime}}$ as functions on $G_{F} \backslash G_{\mathrm{A}} / K^{\prime}$, which is the vertex set of the graph $\mathscr{G}_{\Phi, K^{\prime}}$ of a Hecke operator $\Phi \in \mathscr{H}_{K^{\prime}}$.

We shall investigate the space $\mathscr{A}^{K}$ of unramified automorphic forms in more detail. We write $f(v)$ or $f(\mathcal{M})$ for the value $f(g)$ if $v=[g]$ is the class of $g$ in $G_{F} \backslash G_{\AA} / K$ and $M=M_{g}$ is the rank 2 bundle that corresponds to $g$. In particular, we can see $f$ also as a function on $\mathbb{P B u n}_{2} X$.

The space of automorphic forms decomposes into a cuspidal part $\mathscr{A}_{0}$, a part $\mathscr{E}$ that is generated by Eisenstein series and their derivatives and a part $\mathscr{R}$ that is generated by residues of Eisenstein series and their derivatives (for complete definitions, see [Lorscheid 2010, Section 9.1]). The decomposition descends to unramified automorphic forms: $\mathscr{A}^{K}=\mathscr{A}_{0}^{K} \oplus \mathscr{E}^{K} \oplus \mathscr{R}^{K}$. We describe functions in these parts separately.

9.2. We start with some considerations for $\Phi_{x}$-eigenfunctions as functions on a cusp $\mathscr{C}_{x}(D)$ where $D$ is a divisor with $m_{X}<\operatorname{deg} D \leq m_{X}+d_{x}$ :

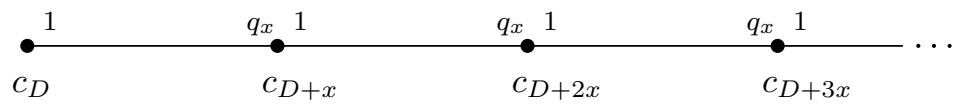

Let $f \in \mathscr{A}^{K}$ satisfy the eigenvalue equation $\Phi_{x} f=\lambda f$, then we obtain for every $i \geq 1$,

$$
f\left(c_{D+(i+1) x}\right)=\lambda f\left(c_{D+i x}\right)-q_{x} f\left(c_{D+(i-1) x}\right) .
$$

Thus the restriction of $f$ to $\operatorname{Vert} \mathscr{C}_{x}(D)$ is determined by the eigenvalue $\lambda$ once its values at $c_{D}$ and $c_{D+x}$ are given. The eigenvalue equation evaluated at $c_{D}$ shows further that $f\left(c_{D+x}\right)$ is a linear combination of values of $f$ in vertices of the nucleus. This consideration justifies that we only have to evaluate the eigenvalue equation at vertices of the nucleus to determine the eigenfunctions of $\Phi_{x}$.

9.3. The space $\mathscr{A}_{0}^{K}$ has a basis of $\mathscr{H}_{K}$-eigenfunctions and every unramified cusp form has a compact, that is, finite, support in $G_{F} \backslash G_{\AA} / K$. By the eigenvalue (9-1) it follows that a Hecke eigenfunction $f \in \mathscr{A}_{0}^{K}$ must vanish on all vertices of a cusp in order to have compact support. Thus the support of a cusp form is contained in the finite set $V$ of vertices $v$ with $\delta(v) \leq m_{X}$, and $\mathscr{A}_{0}^{K}$ can be determined by considering a finite number of eigenvalue equations for $\Phi_{x}$.

These eigenvalue equations can be described in terms of the matrix $M_{x}$ associated with $\Phi_{x}$; see Section 1.8. Namely, $\mathscr{A}_{0}^{K}$ is generated by the eigenfunctions of $M_{x}$ whose support is contained in $V$. This problem can be rephrased into a question on the finite submatrix $M_{x}^{\prime}=\left(a_{v, w}\right)_{v \in V, w \in \operatorname{Vert} \mathcal{N}_{x}}$ of $M_{x}=\left(a_{v, w}\right)_{v, w \in \operatorname{Vert} \varphi_{x}}$, which we forgo spelling out.

In [Moreno 1985] one finds a finite set $S$ of places such that an $\mathscr{H}_{K}$-eigenfunction $f \in \mathscr{A}_{0}^{K}$ is already characterized (up to multiple) by its $\Phi_{x}$-eigenvalues for $x \in S$. 
This means that one finds the cuspidal $\mathscr{H}_{K}$-eigenfunctions by considering the eigenvalue equations for the finitely many vertices $v \in V$ and the finitely many Hecke operators $\Phi_{x}$ for $x \in S$.

9.4. We proceed with $\mathscr{E}^{K} \oplus \mathscr{R}^{K}$. This space decomposes into a direct sum of generalized (infinite-dimensional) Hecke eigenspaces $\mathscr{E}(\chi)$, where $\chi$ runs through all unramified Hecke characters, that is, continuous group homomorphisms

$$
\chi: F^{\times} \backslash \mathbb{A}^{\times} / \mathbb{O}_{\mathbb{A}}^{\times} \rightarrow \mathbb{C}^{\times},
$$

modulo inversion; in particular, $\mathscr{E}(\chi)=\mathscr{E}\left(\chi^{-1}\right)$. The generalized eigenspace $\mathscr{E}(\chi)$ is characterized by its unique Hecke eigenfunction $\tilde{E}(\cdot, \chi)$ (up to scalar multiple), which in turn is determined by its $\Phi_{x}$-eigenvalues $\lambda_{x}(\chi)=q_{x}^{1 / 2}\left(\chi\left(\pi_{x}\right)+\chi^{-1}\left(\pi_{x}\right)\right)$ for $x \in\|X\|$. We have $\mathscr{E}(\chi) \subset \mathscr{E}$ if and only if $\chi^{2} \neq\|\cdot\|^{ \pm 1}$, in which case $\tilde{E}(\cdot, \chi)$ is an Eisenstein series. For $\chi^{2}=\|\cdot\|^{ \pm 1}, \tilde{E}(\cdot, \chi)$ is a residue of an Eisenstein series. For details, see [Lorscheid 2010], in particular, Theorem 11.10.

We say that a subset $S \subset\|X\|$ generates $\mathrm{Cl} X$ if the classes of the prime divisors corresponding to the places in $S$ generate $\mathrm{Cl} X$. Let $S$ be a set of places that generates $\mathrm{Cl} X$ and satisfies that for every decomposition $S=S_{+} \cup S_{-}$either $2 \mathrm{Cl} X=2\left\langle S_{+}\right\rangle$or $2 \mathrm{Cl} X=2\left\langle S_{-}\right\rangle$. This set can be chosen to be finite. Then the Hecke eigenfunction $\tilde{E}(\cdot, \chi)$ is uniquely determined (up to scalar multiples) by the $\Phi_{x}$-eigenvalues $\lambda_{x}(\chi)$. For details, see [Lorscheid 2008, Theorem 3.7.6 and Section 3.7.10].

In order to describe an Eisenstein series or a residue of an Eisenstein series, one only needs to consider the finitely many eigenvalue equations for the vertices in the nuclei $\mathcal{N}_{x}$ of the finitely many Hecke operators $\Phi_{x}$ with $x \in S$. Derivatives of Eisenstein series or residues are similarly determined by generalized eigenvalue equations; see [Lorscheid 2010, Lemmas 11.2 and 11.7] for the explicit formulas.

In the case of a residue, that is, $\chi^{2}=\|\cdot\|^{ \pm 1}$, the function $f=\tilde{E}(\cdot, \chi)$ has a particular simple form. Namely, $\chi$ is of the form $\omega\|\cdot\|^{ \pm 1 / 2}$ where $\omega^{2}=1$ and $\tilde{E}(g, \chi)=\omega \circ \operatorname{det}(g)$. This means that $f\left(g \xi_{w}\right)=\omega\left(\pi_{x} \operatorname{det} g\right)=\omega\left(\pi_{x}\right) f(g)$. Thus, as a function on $\operatorname{Vert} \mathscr{G}, f$ satisfies $f(v)=\omega\left(\pi_{x}\right) f(w)$ for all adjacent vertices $v$ and $w$.

Remark 9.5. The methods of this paragraph will be applied in [Lorscheid 2012] to determine the space of unramified cusp forms for an elliptic function field and to show that there are no unramified toroidal cusp forms in this case.

\section{Finite-dimensionality results}

In this section, we will show how the theory of the last sections can be used to show finite-dimensionality of subspaces of $C^{0}\left(G_{\AA}\right)^{K}$ whose elements $f$ are defined by a 
condition of the form

$$
\sum_{i=1}^{n} m_{i} \Phi(f)\left(g_{i}\right)=0
$$

for all $\Phi \in \mathscr{H}_{K}$ (with $m_{i} \in \mathbb{C}$ and $g_{i} \in G_{\AA}$ being fixed). We will explain a general technique and apply it to show that the spaces of functions in $C^{0}\left(G_{\mathbb{A}}\right)^{K}$ satisfying the cuspidal condition or the toroidal condition, respectively, are finite-dimensional. In particular, this implies that all functions satisfying one of these conditions are automorphic forms.

10.1. Write $\mathrm{Cl}^{\mathrm{pr}} X$ for the set of divisor classes that are represented by prime divisors and $\mathrm{Cl}^{\mathrm{eff}} X$ for the semigroup they generate, that is, for all classes that are represented by effective divisors. In particular, $\mathrm{Cl}^{\mathrm{eff}} X$ contains 0 , the class of the zero divisor, and for all other $[D] \in \mathrm{Cl}^{\text {eff }} X$, we have $\operatorname{deg} D>0$. Denote by $\mathrm{Cl}^{d} X$ the set of divisor classes of degree $d$ and by $\mathrm{Cl}^{\geq} X$ the set of divisor classes of degree at least $d$. Let $g_{X}$ be the genus of $X$.

\section{Lemma 10.2. \\ $\mathrm{Cl}^{\geq g_{X}} X \subset \mathrm{Cl}^{\mathrm{eff}} X$.}

Proof. Let $C$ be a canonical divisor on $X$, which is of degree $2 g_{X}-2$. For a divisor $D$, define $l(D)=\operatorname{dim}_{\mathbb{F}_{q}} H^{0}\left(X, \mathscr{L}_{D}\right)$. We have $[D] \in \mathrm{Cl}^{\text {eff }} X$ if and only if $l(D)>0$; see [Hartshorne 1977, Section IV.1]. The Riemann-Roch theorem is

$$
l(D)-l(D-C)=\operatorname{deg} D+1-g_{X} ;
$$

see [Hartshorne 1977, Theorem IV.1.3].

If now $[D] \in \mathrm{Cl}^{\geq g_{X}} X$, then $\operatorname{deg} D \geq g_{X}$ and the Riemann-Roch theorem implies that $l(D) \geq \operatorname{deg} D+1-g_{X}>0$.

10.3. Let $D$ be an effective divisor. Then it can be written in a unique way up to permutation of terms as a sum of prime divisors $D=x_{1}+\cdots+x_{n}$. We define $\Phi_{0}$ as the identity operator and set $\Phi_{D}=\Phi_{x_{1}} \cdots \Phi_{x_{n}}$. Since $\mathscr{H}_{K}$ is commutative, $\Phi_{D}$ is well-defined. Further we briefly write $\varphi_{D}$ for the graph $\varphi_{\Phi_{D}, K}$ of $\Phi_{D}$, and $u_{D}(v)$ for $u_{\Phi_{D}, K}(v)$.

Let $[D] \in \mathrm{Cl} X$. Recall from Section 5.1 that $\mathscr{L}_{D}$ denotes the associated line bundle and from Section 6.2 that $c_{D}$ denotes the vertex that is represented by $\mathscr{L}_{D} \oplus \mathcal{O}_{X}$. Recall from Proposition 7.7(iv) that $\delta\left(c_{D}\right)=\|\operatorname{deg} D\|$, where $\delta$ is defined as in Section 7.1.

Lemma 10.4. Let $D$ be an effective divisor.

(i) Let $v, v^{\prime} \in \operatorname{Vert} \varphi_{D}$. If $v^{\prime}$ is a $\Phi_{D}$-neighbor of $v$, then $\left\|\delta\left(v^{\prime}\right)-\delta(v)\right\| \leq \operatorname{deg} D$.

(ii) Let $[\mathcal{M}] \in \operatorname{Vert} \mathscr{G}_{D}$. Every maximal subbundle $\mathscr{L} \rightarrow \mathcal{M}$ lifts to a maximal subbundle $\mathscr{L} \rightarrow \mathcal{M}^{\prime}$ of a uniquely determined rank 2 bundle $\mathcal{M}^{\prime}$ such that $\left[\mathcal{M}^{\prime}\right]$ is a $\Phi_{D}$-neighbor of $[\mathcal{M}]$ with $\delta\left(\mathcal{M}^{\prime}\right)=\delta(\mathcal{M})+\operatorname{deg} D$. Conversely, every 
maximal subbundle $\mathscr{L} \rightarrow \mathcal{M}^{\prime}$ extends to a maximal subbundle $\mathscr{L} \rightarrow \mathcal{M}$ if $\left[\mathcal{M}^{\prime}\right]$ is a $\Phi_{D}$-neighbor of $[M]$ with $\delta\left(\mathcal{M}^{\prime}\right)=\delta(\mathcal{M})+\operatorname{deg} D$.

Proof. We do induction on the number of factors in $\Phi_{D}=\Phi_{x_{1}} \cdots \Phi_{x_{n}}$ with $x_{1}, \ldots, x_{n}$ being prime divisors. The lemma is trivial for the identity operator $\Phi_{0}$.

If $n \geq 1$, write $x=x_{n}$ and $\Phi_{D}=\Phi_{D^{\prime}} \Phi_{x}$ for the effective divisor

$$
D^{\prime}=x_{1}+\cdots+x_{n-1},
$$

which is of degree $\operatorname{deg} D^{\prime}=\operatorname{deg} D-\operatorname{deg} x$. Assume that (i) and (ii) hold for $D^{\prime}$. Let $v^{\prime}$ be a $\Phi_{D^{-}}$-neighbor of $v$. Let $m$ be the weight of the edge $\left(v, v^{\prime}, m\right)$. As explained in Section 1.7, we have

$$
\sum_{\substack{\left(v, v^{\prime \prime}, m^{\prime}\right) \in \operatorname{Edge} \mathscr{G}_{D^{\prime}} \\\left(v^{\prime \prime}, v^{\prime}, m^{\prime \prime}\right) \in \operatorname{Edge} \varphi_{x}}} m^{\prime} \cdot m^{\prime \prime}=m \neq 0,
$$

which means that there is a $v^{\prime \prime}$ that is a $\Phi_{D^{\prime}}$-neighbor of $v$ and a $\Phi_{x}$-neighbor of $v^{\prime}$. Thus the inductive hypothesis and Lemma 8.2 imply

$$
\left\|\delta\left(v^{\prime}\right)-\delta(v)\right\| \leq\left\|\delta\left(v^{\prime}\right)-\delta\left(v^{\prime \prime}\right)\right\|+\left\|\delta\left(v^{\prime \prime}\right)-\delta(v)\right\| \leq \operatorname{deg} D^{\prime}+\operatorname{deg} x=\operatorname{deg} D .
$$

This proves (i).

We proceed with (ii). Let $\mathscr{L} \rightarrow \mathcal{M}$ be a maximal subbundle. By the inductive hypothesis, there is a $\Phi_{D^{\prime}}$-neighbor $\mathcal{M}^{\prime \prime}$ of $\mathcal{M}$ such that $\mathscr{L} \rightarrow \mathcal{M}$ lifts to a maximal subbundle of $\mathcal{M}^{\prime \prime}$ and such that $\delta\left(\mathcal{M}^{\prime \prime}\right)=\delta(\mathcal{M})+\operatorname{deg} D^{\prime}$. Let

$$
0 \rightarrow \mathcal{M}^{\prime} \rightarrow \mathcal{M}^{\prime \prime} \rightarrow \mathscr{K}_{x} \rightarrow 0
$$

be the sequence associated with $\mathscr{L} \rightarrow \mathcal{M}^{\prime \prime}$. This means that $\mathscr{L}$ lifts to a subbundle of $\mathcal{M}^{\prime}$. As explained in Section $8.3, \delta\left(\mathscr{L}, \mathcal{M}^{\prime}\right)=\delta\left(\mathscr{L}, \mathcal{M}^{\prime \prime}\right)+\operatorname{deg} x$, where $\delta\left(\mathscr{L}, \mathcal{M}^{\prime \prime}\right)=\delta\left(\mathcal{M}^{\prime \prime}\right)$ by the maximality of $\mathscr{L}$. By part (i) of the lemma, we have $\delta\left(\mathcal{M}^{\prime}\right) \leq \delta\left(\mathcal{M}^{\prime \prime}\right)+\operatorname{deg} x=\delta\left(\mathscr{L}, \mathcal{M}^{\prime}\right)$, which must be an equality in this case. Therefore $\mathscr{L} \rightarrow \mathcal{M}^{\prime}$ is maximal and

$$
\delta\left(\mathcal{M}^{\prime}\right)=\delta\left(\mathcal{M}^{\prime \prime}\right)+\operatorname{deg} x=\delta(\mathcal{M})+\operatorname{deg} D^{\prime}+\operatorname{deg} x=\delta(\mathcal{M})+\operatorname{deg} D,
$$

as desired.

Assume conversely that $\mathcal{M}^{\prime}$ is a $\Phi_{D}$-neighbor of $\mathcal{M}^{\prime}$ with $\delta\left(\mathcal{M}^{\prime}\right)=\delta(\mathcal{M})+\operatorname{deg} D$ and let $\mathscr{L} \rightarrow \mathcal{M}^{\prime}$ be a maximal subbundle. As already explained in the proof of (i), there is an $\mathcal{M}^{\prime \prime}$, which is a $\Phi_{D^{\prime}}$-neighbor of $\mathcal{M}^{\prime}$ and a $\Phi_{x}$-neighbor of $\mathcal{M}$. By (i), the difference of $\delta(M)$ and $\delta\left(\mathcal{M}^{\prime}\right)$ is maximal; therefore it must hold that $\delta\left(\mathcal{M}^{\prime}\right)=\delta\left(\mathcal{M}^{\prime \prime}\right)+\operatorname{deg} D^{\prime}$ and $\delta\left(\mathcal{M}^{\prime \prime}\right)=\delta(\mathcal{M})+\operatorname{deg} x$. By the inductive hypothesis, $\mathscr{L} \rightarrow \mathcal{M}^{\prime \prime}$ is a maximal subbundle, that is, $\delta\left(\mathcal{M}^{\prime \prime}\right)=\delta\left(\mathscr{L}, \mathcal{M}^{\prime \prime}\right)$. We derive $\delta\left(\mathcal{M}^{\prime \prime}\right)=\delta(\mathcal{M})+\operatorname{deg} x \geq 2 \operatorname{deg} \mathscr{L}-\operatorname{deg} \mathcal{M}+\operatorname{deg} x=2 \operatorname{deg} \mathscr{L}-\operatorname{deg} \mathcal{M}^{\prime \prime}=\delta\left(\mathcal{M}^{\prime \prime}\right)$. 
Consequently, all inequalities are equalities and $\mathscr{L} \rightarrow \mathcal{M}$ is a maximal subbundle, what was to be shown.

10.5. We demonstrate how to use the lemma to show that the space $\mathscr{V}_{0}$ of all unramified functions on $G_{F} \backslash G_{\mathbb{A}}$ that satisfy the cuspidal condition is finitedimensional. Namely, let $N \subset G$ be a unipotent subgroup. Then the cuspidal condition for $f \in C^{0}\left(G_{F} \backslash G_{\mathbb{A}}\right)^{K}$ is that

$$
\int_{N_{F} \backslash N_{\AA}} \Phi(f)(n) d n=0 \quad \text { for all } \Phi \in \mathcal{H} .
$$

If $f$ is an automorphic form, then this condition defines a cusp form. A posteriori it will be clear that $\mathscr{V}_{0}$ contains only automorphic forms and thus equals the space $\mathscr{A}_{0}^{K}$ of unramified cusp forms.

Theorem 10.6. The dimension of $\mathscr{V}_{0}$ is finite and bounded by

$$
\operatorname{dim} \mathscr{V}_{0} \leq \#\left\{[\mathcal{M}] \in \mathbb{P B u n} 2 X \mid \delta(\mathcal{M}) \leq m_{X}\right\} .
$$

Proof. Note that there are only finitely many projective line bundles $[M]$ with $\delta(M) \leq m_{X}$ since $\mathbb{P B u n}{ }_{2}^{\text {indec }} X$ is finite and $\mathbb{P B u n}_{2}^{\text {dec }} X$ has only finitely many classes $[\mathcal{M}]$ with $\delta(\mathcal{M}) \leq m_{X}$. So the finite-dimensionality of $\mathscr{V}_{0}$ will follow from the inequality.

We proceed with the proof of the inequality. The geometric equivalent of the cuspidal condition is that

$$
\sum_{\mathcal{M} \in \operatorname{Ext}^{1}\left(\mathscr{O}_{X}, \mathscr{O}_{X}\right)} \Phi(f)(\mathcal{M})=0 \quad \text { for all } \Phi \in \mathscr{H} ;
$$

see [Gaitsgory 2003].

Since $\delta\left(\mathscr{O}_{X}, \mu\right)=0$ for $M \in \operatorname{Ext}^{1}\left(O_{X}, \mathscr{O}_{X}\right)$, we have that $\mathscr{O}_{X} \rightarrow \mathcal{M}$ is a maximal subbundle by Proposition 7.7(ii), and only in the case of the trivial extension $\mathcal{M} \simeq O_{X} \oplus O_{X}$ are there other maximal subbundles, namely, there exist $(q+1)$ different subbundles of the form $\mathrm{O}_{X} \rightarrow \mathcal{M}$. Note that in any case, $\delta(\mathcal{M})=0$.

Let $D$ be a nontrivial effective divisor. In case $M$ is the trivial extension $\mathscr{O}_{X} \oplus \mathcal{O}_{X}$, the vertex $c_{0}=[\mathcal{M}]$ has the unique $\Phi_{D}$-neighbor $v^{\prime}=c_{D}$ with $\delta\left(v^{\prime}\right)=\operatorname{deg} D$, which is of multiplicity $q+1$, as follows from an easy induction using Theorem 8.5 and Lemma 10.4. In case $M$ is a nontrivial extension of $O_{X}$ by itself, the vertex $v=[M]$ has a unique $\Phi_{D}$-neighbor $v^{\prime}=\left[\mathcal{M}^{\prime}\right]$ with $\delta\left(v^{\prime}\right)-\delta(v)=\operatorname{deg} D$, which has a unique maximal subbundle, namely, $\mathrm{O}_{X} \rightarrow \mathcal{M}^{\prime}$.

Thus for every $M \in \operatorname{Ext}^{1}\left(\mathcal{O}_{X}, \mathscr{O}_{X}\right)$ and every $\Phi_{D}$-neighbor [ $\left.\mathcal{M}^{\prime}\right]$ of $[M]$ with $\delta\left(\mathcal{M}^{\prime}\right)=\operatorname{deg} D$, the maximal subbundles of $\mathcal{M}^{\prime}$ are of the form $O_{X} \rightarrow \mathcal{M}^{\prime}$. Thus if $\operatorname{deg} D>m_{X}$, then $\mathcal{M}^{\prime} \simeq \mathfrak{O}_{X} \oplus\left(\mathcal{M}^{\prime} / \mathscr{O}_{X}\right)$ by Proposition 7.2. Since the determinant is multiplicative and $\operatorname{det} \mathscr{K}_{x} \simeq \mathscr{L}_{x}$ (see [Hartshorne 1977, Ex. 6.11]), a short exact 
sequence $0 \rightarrow M_{1} \rightarrow M_{2} \rightarrow \mathscr{K}_{x} \rightarrow 0$ yields $\operatorname{det} \mathcal{M}_{2} \simeq \mathscr{L}_{x} \otimes \operatorname{det} \mathcal{M}_{1}$. An easy induction over the length of the prime decomposition $D=x_{1}+\cdots+x_{n}$ shows that $\operatorname{det} \mathcal{M} \simeq \mathscr{L}_{D} \otimes \operatorname{det} \mathcal{M}^{\prime}$. Therefore we have $\mathcal{M}^{\prime} / \mathcal{O}_{X} \simeq \mathscr{L}_{-D}$, which shows that $\left[\mathcal{M}^{\prime}\right]=c_{D}$.

We finish the proof of the theorem by showing that every $f \in \mathscr{V}_{0}$ is determined by its values in the vertices $v$ with $\delta(v) \leq m_{X}$. We make an induction on $d=\delta\left(c_{D}\right)$, where $c_{D}$ varies through all vertices $v$ with $\delta(v)>m_{X}$.

Let $d>m_{X}$. Assume that the values of $f$ in all vertices $v$ with $\delta(v)<d$ are given (which is the case when $d=m_{X}+1$; thus the initial step). Let $v$ be a vertex with $\delta(v)=d$. Then $v=c_{D}$ for an effective divisor $D$ by Lemma 10.2 since $m_{X}=\max \left\{0,2 g_{X}-2\right\} \geq g_{X}-1$. For the Hecke operator $\Phi_{D}$, the cuspidal condition reads by the previous argumentation and Lemma 10.4 as

$$
\left(q+q^{e_{1}}\right) \cdot f\left(c_{D}\right)+\sum_{\delta\left(v^{\prime}\right)<d} a_{v^{\prime}} f\left(v^{\prime}\right)=0
$$

for certain $a_{v^{\prime}}$ and $e_{1}=\operatorname{dim} \operatorname{Ext}^{1}\left(O_{X}, O_{X}\right)$. Thus $f(v)$ is determined by the values $f\left(v^{\prime}\right)$ in vertices $v^{\prime}$ with $\delta\left(v^{\prime}\right)<d$, which proves the theorem.

10.7. While the finite-dimensionality of $\mathscr{V}_{0}$ can also be established without the techniques of this paper, we do not know any other method to prove the corresponding fact for toroidal functions. For more details on the following definitions, see [Lorscheid 2010].

Choose a basis of $\mathbb{F}_{q^{2}}$ over $\mathbb{F}_{q}$. This defines an embedding of $E=\mathbb{F}_{q^{2}} F$ into the algebra of $2 \times 2$-matrices with entries in $F$. The image of $E^{\times}$is contained in $\mathrm{GL}_{2}(F)$ and defines a nonsplit torus $T^{\prime}$ of $\mathrm{GL}_{2}$. The image of $T^{\prime}$ in $G=\mathrm{GL}_{2} / Z$ defines a nonsplit torus $T$ of $G$.

A function $f \in C^{0}\left(G_{F} \backslash G_{\AA}\right)^{K}$ is E-toroidal if for all $\Phi \in \mathscr{H}_{K}$,

$$
\int_{T_{F} \backslash T_{\AA}} \Phi(f)(t) d t=0 .
$$

We denote the space of all $E$-toroidal functions $f \in C^{0}\left(G_{F} \backslash G_{\mathrm{A}}\right)^{K}$ by $\mathscr{V}_{\text {tor }}$. Note that in [Lorscheid 2010] one finds a toroidal condition that is stronger than $E$-toroidality. Namely, $f$ has to be $E^{\prime}$-toroidal for all separable quadratic algebra extensions $E^{\prime}$ of $F$. We forgo recalling complete definitions, but remark that the finite-dimensionality of the space of all toroidal $f \in C^{0}\left(G_{F} \backslash G_{\AA}\right)^{K}$ follows since it is a subspace of $\mathscr{V}_{\text {tor }}$. Let $p: X^{\prime} \rightarrow X$ be the map of curves that corresponds to the field extension $E / F$. 
Theorem 10.8. Let $c_{T}=\operatorname{vol}\left(T_{F} \backslash T_{\mathbb{A}}\right) / \#\left(\operatorname{Pic} X^{\prime} / p^{*}(\operatorname{Pic} X)\right)$. Then,

$$
\int_{T_{F} \backslash T_{\AA}} f(t) d t=c_{T} \cdot \sum_{[\mathscr{L}] \in \operatorname{Pic} X^{\prime} / p^{*}(\operatorname{Pic} X)} f\left(\left[p_{*} \mathscr{L}\right]\right) \quad \text { for all } f \in C^{0}\left(G_{F} \backslash G_{\AA}\right)^{K} .
$$

Proof. Let $\mathbb{A}_{E}$ be the adèles of $E$. To avoid confusion, we write $\mathbb{A}_{F}$ for $\mathbb{A}$. We introduce the following notation. For an $x \in\|X\|$ that is inert in $E / F$, we define $\mathcal{O}_{E, x}:=\mathfrak{O}_{E, y}$, where $y$ is the unique place that lies over $x$. For an $x \in\|X\|$ that is split in $E / F$, we define $\mathscr{O}_{E, x}:=\mathscr{O}_{E, y_{1}} \oplus \mathcal{O}_{E, y_{2}}$, where $y_{1}$ and $y_{2}$ are the two places that lie over $x$. Note that there is no place that ramifies. Let $O_{E_{x}}$ denote the completion of $O_{E, x}$. Then $\mathcal{O}_{E_{x}}$ is a free module of rank 2 over $\mathcal{O}_{F_{x}}=\mathcal{O}_{x}$ for every $x \in\|X\|$.

Let $\Theta_{E}: \mathbb{A}_{E}^{\times} \rightarrow \mathrm{GL}_{2}\left(\mathbb{A}_{F}\right)$ be the base extension of the embedding $E^{\times} \rightarrow \mathrm{GL}_{2}(F)$ that defines $T^{\prime}$, which corresponds to the chosen basis of $E$ over $F$ that is contained in $\mathbb{F}_{q^{2}}$. This basis is also a basis of $\mathbb{O}_{E_{x}}$ over $\mathscr{O}_{F_{x}}$ for every $x \in\|X\|$. This shows that $\Theta_{E}^{-1}\left(\operatorname{GL}_{2}\left(\mathcal{O}_{\mathrm{A}_{F}}\right)\right)=\mathcal{O}_{\mathrm{A}_{E}}^{\times}$and that the diagram

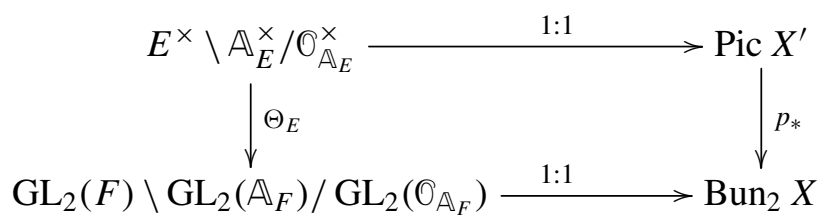

commutes, where the horizontal arrows are the bijections defined in Section 5.3.

The action of $\mathbb{A}_{F}$ on $E^{\times} \backslash \mathbb{A}_{E}^{\times} / \mathbb{O}_{\mathbb{A}_{E}}^{\times}$and $\mathrm{GL}_{2}(F) \backslash \mathrm{GL}_{2}\left(\mathbb{A}_{F}\right) / \mathrm{GL}_{2}\left(\mathrm{O}_{\mathbb{A}_{F}}\right)$ by scalar multiplication is compatible with the action of Pic $X$ on Pic $X^{\prime}$ and $\operatorname{Bun}_{2} X$ by tensoring in the sense that all maps in the diagram above are equivariant if we identify Pic $X$ with $F^{\times} \backslash \mathbb{A}_{F}^{\times} / \mathcal{O}_{\mathbb{A}_{F}}^{\times}$. Taking orbits under these compatible actions yields the commutative diagram

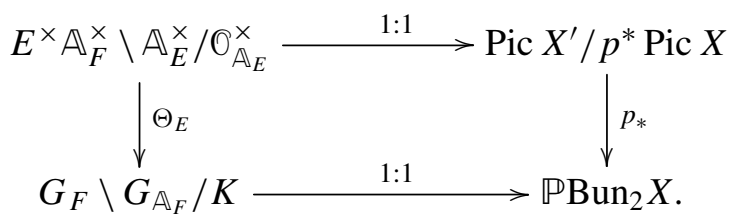

Since $f$ is right $K$-invariant, we may take the quotient of the domain of integration by $T_{\mathbb{A}_{F}} \cap K$ from the right, which is the image of $O_{A_{E}}^{\times}$in $G_{\mathbb{A}_{F}}$. We obtain the assertion of the theorem for some still undetermined value of $c$. The value of $c$ is computed by plugging in a constant function for $f$.

Theorem 10.9. The space of unramified toroidal functions has finite dimension, bounded by

$$
\operatorname{dim} \mathscr{V}_{\text {tor }} \leq \#\left(\mathbb{P B u n} \text { B }_{2} X-\left\{c_{D}\right\}_{\left.[D] \in \mathrm{Cl}\right|^{\text {eff }} X}\right)
$$


Proof. Given the inequality in the theorem, finite-dimensionality follows since the right-hand set is finite. Indeed, by Lemma 10.2,

$$
\mathbb{P B u n}_{2} X-\left\{c_{D}\right\}_{[D] \in \mathrm{Cl}^{\text {eff }} X} \subset\left\{v \in \mathbb{P B u n} 2 X \mid \delta(v) \leq m_{X}\right\}
$$

since $m_{X} \geq g_{X}-1$, and the latter set is finite.

We now proceed with the proof of the inequality. Let $f \in \mathscr{V}_{\text {tor }}$. We will show by induction on $d=\operatorname{deg} D$ that the value of $f$ at a vertex $c_{D}$ with $[D] \in \mathrm{Cl}^{\text {eff }} X$ is uniquely determined by the values of $f$ at the elements of $\mathbb{P B u n}_{2} X-\left\{c_{D}\right\}_{[D] \in \mathrm{Cl}^{\text {eff }} X}$. This will prove the theorem.

By Theorem 10.8, the condition for $f$ to lie in $\mathscr{V}_{\text {tor }}$ reads

$$
\sum_{[\mathscr{L}] \in\left(\operatorname{Pic} X^{\prime} / p^{*} \operatorname{Pic} X\right)} \Phi(f)\left(\left[p_{*} \mathscr{L}\right]\right)=0 \quad \text { for all } \Phi \in \mathscr{H} .
$$

If $d=0$, take $\Phi$ as the identity element in $\mathscr{H}_{K}$. We know from Proposition 6.4 that $p_{*}\left(\operatorname{Pic} X^{\prime} / p^{*} \operatorname{Pic} X\right)=\mathbb{P B u n}{ }_{2}^{\text {tr }} X \cup\left\{c_{0}\right\}$, so $f\left(c_{0}\right)$ equals a linear combination of values of $f$ at vertices $v$ in $\mathbb{P B u n}{ }_{2}^{\mathrm{tr}} X$, which all satisfy $\delta(v)<0$ by Proposition 7.8. Since the zero divisor class is the only class in $\mathrm{Cl}^{\text {eff }} X$ of degree 0 , we have proven the case $d=0$.

Next, let $D$ be an effective divisor of degree $d>0$ and put $\Phi=\Phi_{D}$. If $v$ is a $\Phi_{D}$-neighbor of $w$, then $\delta(v)$ and $\delta(w)$ can differ at most by $d$ (Lemma 10.4(i)). Therefore all $\Phi_{D}$-neighbors $v$ of vertices in $\operatorname{PBun}_{2}^{\text {tr }} X$ have $\delta(v)<d$. The vertex $c_{D}$ is the only $\Phi_{D}$-neighbor $v$ of $c_{0}$ with $\delta(v)=d$ (as already seen in the proof of Theorem 10.6). Thus

$$
0=\sum_{\mathscr{L} \in\left(\operatorname{Pic} X^{\prime} / p^{*} \operatorname{Pic} X\right)} \Phi_{D}(f)\left(\left[p_{*} \mathscr{L}\right]\right)=(q+1) f\left(c_{D}\right)+\sum_{\substack{\mathscr{L} \in\left(\operatorname{Pic} X^{\prime} / p^{*} \operatorname{Pic} X\right),\left(\left[p_{*} \mathscr{L}\right], v, \lambda\right) \in \mathscr{U}_{D}\left(\left[p_{*} \mathscr{L}\right]\right), \delta(v)<d}} \lambda f(v)
$$

determines $f\left(c_{D}\right)$ as the linear combination of values of $f$ at vertices $v$ satisfying $\delta(v)<d$. By the inductive hypothesis, $f\left(c_{D}\right)$ is already determined by the values of $f$ at vertices that are not contained in $\left\{c_{D}\right\}_{[D] \in \mathrm{Cl}^{\text {fff }} X}$.

Example 10.10. If $X$ is the projective line over $\mathbb{F}_{q}$, then all vertices $v$ are of the form $c_{D}$ for some effective divisor $D$ (see Example 8.10). Thus $\mathscr{V}_{\text {tor }}$ is trivial. Since only $v=c_{0}$ satisfies $\delta(v) \leq m_{X}$, all values of $f \in \mathscr{V}_{0}$ are multiples of $f\left(c_{0}\right)$. However, $\operatorname{Ext}^{1}\left(\mathcal{O}_{X}, O_{X}\right)$ is trivial, thus the cuspidal condition (applied to the trivial Hecke operator) is $f\left(c_{0}\right)=0$. Thus also $\mathscr{V}_{0}$ is trivial. See [Lorscheid 2012] for the corresponding spaces in the case of an elliptic curve. 


\section{Appendix: Examples for rational function fields}

We give examples of graphs of Hecke operators for a rational function field, which can be calculated by elementary matrix manipulations. We do not show all calculations, but hint on how to do them. The reader will find examples for elliptic function fields that are determined by geometric methods in [Lorscheid 2012].

Let $F$ be $\mathbb{F}_{q}(T)$, the function field of the projective line over $\mathbb{F}_{q}$, which has $q+1$ $\mathbb{F}_{q}$-rational points and trivial class group. Fix a place $x$ of degree 1 .

A.1. Using strong approximation for $\mathrm{SL}_{2}$ (see Proposition 3.8, where $J$ is trivial in this case), we get a bijection by adding the identity matrix $e$ at all places $y \neq x$ :

$$
\Gamma \backslash G_{x} / K_{x} \rightarrow G_{F} \backslash G_{\mathbb{A}} / K, \quad\left[g_{x}\right] \mapsto\left[\left(g_{x}, e\right)\right] .
$$

We introduce some notation. Elements of $\mathcal{O}_{F}^{x}=\mathcal{O}^{x} \cap F$ can be written in the form $\sum_{i=m}^{0} b_{i} \pi_{x}^{i}$ with $b_{i} \in \mathbb{F}_{q}$ for $i=m, \ldots, 0$ for some integer $m \leq 0$. Let $\tilde{K}_{x}=\mathrm{GL}_{2}\left(\mathrm{O}_{x}\right)$, where we view $\mathcal{O}_{x}$ as the collection of all power series $\sum_{i \geq 0} b_{i} \pi_{x}^{i}$ with $b_{i} \in \mathbb{F}_{q}$ for $i \geq 0$. Let $\Gamma=\mathrm{GL}_{2}\left(\mathcal{O}_{F}^{x}\right)$ and let $Z$ be the center of $\mathrm{GL}_{2}$.

A.2. For better readability, we write $\pi$ for the uniformizer $\pi_{x}$ at $x$ and $g$ for a matrix in $G_{x}$. We say $g \sim g^{\prime}$ if they represent the same class $[g]=\left[g^{\prime}\right]$ in $\Gamma \backslash G_{x} / K_{x}$, and indicate by subscripts to " $\sim$ " how to alter one representative to another. The following changes of the representative $g$ of a class $[g] \in \Gamma \backslash G_{x} / K_{x}$ provide an algorithm to determine a standard representative for the class of any matrix $g \in G_{x}$ :

(i) By the Iwasawa decomposition, every class in $\Gamma \backslash G_{x} / K_{x}$ is represented by an upper triangular matrix, and

$$
\left(\begin{array}{ll}
a & b \\
& d
\end{array}\right) \underset{/ Z_{x}}{\sim}\left(\begin{array}{ll}
a & b \\
& d
\end{array}\right)\left(\begin{array}{ll}
d^{-1} & \\
& d^{-1}
\end{array}\right)=\left(\begin{array}{cc}
a / d & b / d \\
& 1
\end{array}\right)
$$

(ii) Write $a / d=r \pi^{n}$ for some integer $n$ and $r \in \mathcal{O}_{x}^{\times}$, then with $b^{\prime}=b / d$, we have

$$
\left(\begin{array}{rr}
r \pi^{n} & b^{\prime} \\
& 1
\end{array}\right) \underset{/ \tilde{K}_{x}}{\sim}\left(\begin{array}{rr}
r \pi^{n} & b^{\prime} \\
& 1
\end{array}\right)\left(\begin{array}{ll}
r^{-1} & \\
& 1
\end{array}\right)=\left(\begin{array}{cc}
\pi^{n} & b^{\prime} \\
& 1
\end{array}\right) .
$$

(iii) If $b^{\prime}=\sum_{i \geq m} b_{i} \pi^{i}$ for some integer $m$ and coefficients $b_{i} \in \mathbb{F}_{q}$ for $i \geq m$, then

$$
\begin{aligned}
& \left(\begin{array}{cc}
\pi^{n} & \sum_{i \geq m} b_{i} \pi^{i} \\
& 1
\end{array}\right) \underset{/ \tilde{K}_{x}}{\sim}\left(\begin{array}{cc}
\pi^{n} & \sum_{i \geq m} b_{i} \pi^{i} \\
& 1
\end{array}\right)\left(\begin{array}{cc}
1 & -\pi^{-n}\left(\sum_{i \geq n} b_{i} \pi^{i}\right) \\
1 & 1
\end{array}\right) \\
& =\left(\begin{array}{cc}
\pi^{n} & b_{m} \pi+\cdots+b_{n-1} \pi^{n-1} \\
1 & 1
\end{array}\right) \text {. }
\end{aligned}
$$


(iv) One can further perform the following step:

$$
\begin{aligned}
& \left(\begin{array}{cc}
\pi^{n} & b_{m} \pi^{m}+\cdots+b_{n-1} \pi^{n-1} \\
1
\end{array}\right) \\
& \widetilde{\Gamma \backslash}\left(\begin{array}{c}
1-\left(b_{m} \pi^{m}+\cdots+b_{0} \pi^{0}\right) \\
1
\end{array}\right)\left(\begin{array}{cc}
\pi^{n} & b_{m} \pi^{m}+\cdots+b_{n-1} \pi^{n-1} \\
1
\end{array}\right) \\
& =\left(\begin{array}{cc}
\pi^{n} & b_{1} \pi+\cdots+b_{n-1} \pi^{n-1} \\
1
\end{array}\right) \text {. }
\end{aligned}
$$

(v) If $b=b_{1} \pi+\cdots+b_{n-1} \pi^{n-1} \neq 0$, then $b=s \pi^{k}$ with $1 \leq k \leq n-1, s \in O_{x}^{\times}$ and

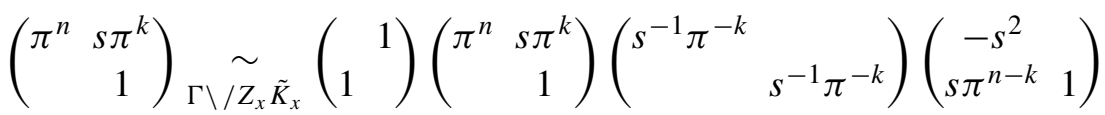

$$
\begin{aligned}
& =\left(\begin{array}{cc}
\pi^{n-2 k} & s^{-1} \pi^{-k} \\
& 1
\end{array}\right) \text {. }
\end{aligned}
$$

(vi) The last trick is

$$
\left(\begin{array}{ll}
\pi^{n} & \\
& 1
\end{array}\right) \underset{\Gamma \backslash / Z_{x} \tilde{K}_{x}}{\sim}\left(\begin{array}{ll}
1 & 1
\end{array}\right)\left(\begin{array}{ll}
\pi^{n} & \\
& 1
\end{array}\right)\left(\begin{array}{cc}
\pi^{-n} & \\
& \pi^{-n}
\end{array}\right)\left(\begin{array}{ll}
1 \\
1 &
\end{array}\right)=\left(\begin{array}{ll}
\pi^{-n} & \\
& 1
\end{array}\right) .
$$

Executing these steps (possibly (iii)-(v) several times) will finally lead to a matrix of the form $p_{n}=\operatorname{diag}\left(\pi^{-n}, 1\right)$ for some $n \geq 0$. The matrix $p_{n}$ represents the vertex $c_{n x}$ in $\operatorname{Vert} \mathscr{G}_{\Phi, K}=\left\{c_{n x}\right\}_{n \geq 0}$ where $\Phi$ is any unramified Hecke operator (see Example 8.10). Thus we found a way to determine the vertex $c_{n x}$ represented by an arbitrary matrix $g \in G_{x} \subset G_{\mathrm{A}}$.

Example A.3 (graph of 0 and 1). According to Section 1.7, the graph for the zero element 0 in $\mathscr{H}_{K}$ is

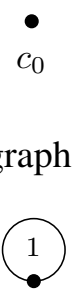

$c_{0}$

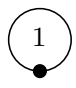

$c_{x}$

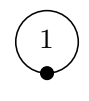

$c_{2 x}$

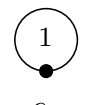

$c_{3 x}$

Example A.4 (graph of $\Phi_{x}$ ). By Proposition 2.3, the $\Phi_{x}$-neighbors of $p_{i}$ are of the form $p_{i} \xi_{w}$. With help of the reduction steps (i)-(vi) in A.2 one can determine easily the standard representative $p_{j}$ of $p_{i} \xi_{w}$. We reobtain the graph of $\Phi_{x}$ as illustrated 


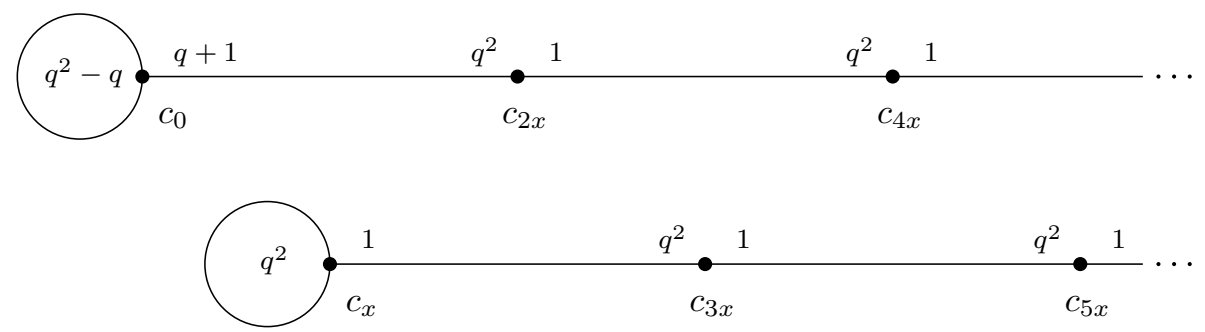

Figure A1. The graph of $\Phi_{y}$ for a place $y$ of degree 2 .

below (compare with Example 8.10).

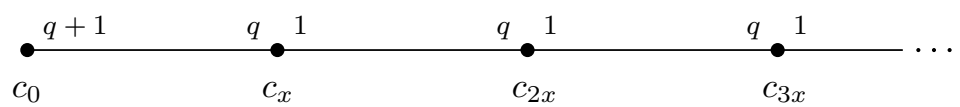

Example A.5 (graph of $\Phi_{y}$ for $y \neq x$ ). If we want to determine the edges of $\varphi_{y}$ for a place $y$ of degree $d$ that differs from $x$, we have to find the standard representative $p_{j}$ for each of the elements $p_{i} \xi_{w}$ where $w \in \mathbb{P}^{1}\left(\kappa_{y}\right)$, that is, $\xi_{w}$ is an element of the form

$$
\left(\begin{array}{ll}
\pi_{y} & b \\
& 1
\end{array}\right) \quad \text { with } b \in \kappa_{y}, \quad \text { or }\left(\begin{array}{ll}
1 & \\
& \pi_{y}
\end{array}\right) \text {. }
$$

Since the class number of $F$ is 1 , the strong approximation property yields $G_{F} K^{x}=$ $G_{\AA}^{x}$ (see Proposition 3.8). This means that we find elements $\gamma \in G_{F}$ and $k \in K$ such that for all $z \neq x$, the adelic matrices $\xi_{w}$ and $\gamma k$ have equal $z$-components $\left(\xi_{w}\right)_{z}=(\gamma k)_{z}$. Therefore, the only nontrivial component of the adelic matrix

$$
\theta_{w}=\gamma^{-1} \xi_{w} k^{-1}
$$

is its $x$-component. By an appropriate choice of $k_{x}$, we can normalize the $x$-component of $\theta_{w}$ to be equal to one of the matrices

$$
\left(\begin{array}{cc}
\pi_{x}^{d} & b_{0}+\cdots+b_{d-1} \pi_{x}^{d-1} \\
& 1
\end{array}\right) \text { with } b_{i} \in \kappa_{x} \text { for } i=0, \ldots, d-1, \quad \text { and }\left(\begin{array}{cc}
1 & \\
& \pi_{x}^{d}
\end{array}\right) \text {, }
$$

and for the different choices of $w \in \mathbb{P}^{1}\left(\kappa_{y}\right)$, each of these matrices occurs as the $x$-component of a (unique) $\theta_{w}$. The reduction steps (i)-(vi) of A.2 tell us which classes $p_{j}$ are represented by the matrices $\theta_{w} p_{i}=\gamma^{-1} p_{i} \xi_{w} k^{-1}$, and we are able to determine the edges similarly to the previous example. Thus we obtain that $\varphi_{y}$ only depends on the degree of $y$. Note that if $y$ is of degree 1 , then $\mathscr{G}_{y}$ equals $\mathscr{G}_{x}$. Figures A1, A2, A5, and A6 show the graphs for degrees 2, 3, 4 and 5, respectively.

Example A.6 (the graph of powers of $\Phi_{x}$ ). It is interesting to compare the graph of $\Phi_{y}$ with deg $y=d$ to the graph of $\Phi_{x}^{d}$. The latter graph is easily deduced from $\mathscr{G}_{x}$ by 


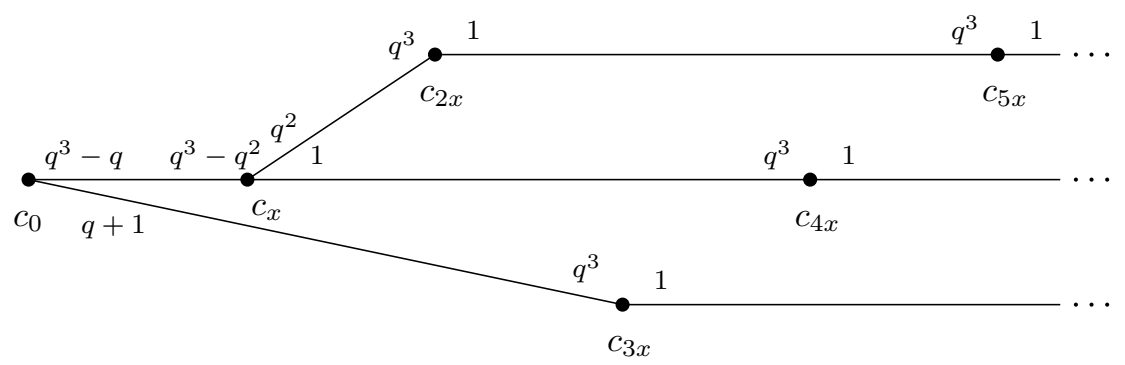

Figure A2. The graph of $\Phi_{y}$ for a place $y$ of degree 3 .

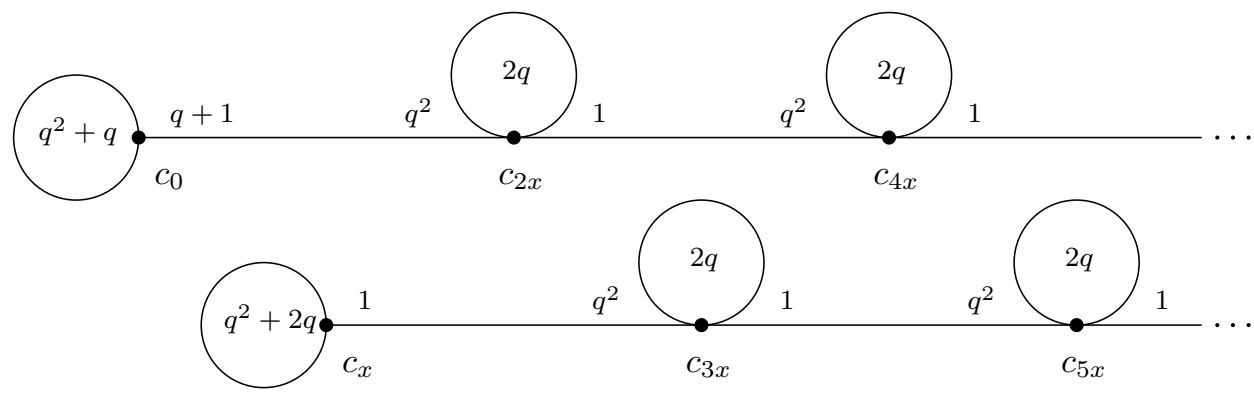

Figure A3. The graph of $\Phi_{x}^{2}$.

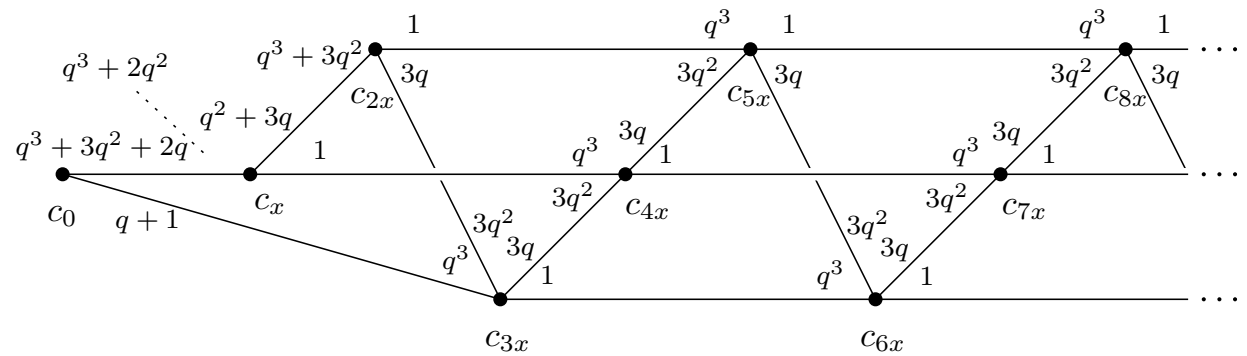

Figure A4. The graph of $\Phi_{x}^{3}$.

means of Section 1.7. Namely, a vertex $v^{\prime}$ is a $\Phi_{x}^{d}$-neighbor of a vertex $v$ in $\mathscr{G}_{\Phi_{x}^{d}, K}$ if there is a path of length $d$ from $v$ to $v^{\prime}$ in $\mathscr{G}_{x}$, that is, a sequence $\left(v_{0}, v_{1}, \ldots, v_{d}\right)$ of vertices in $\varphi_{x}$ with $v_{0}=v$ and $v_{d}=v^{\prime}$ such that for all $i=1, \ldots, d$, there is an edge $\left(v_{i-1}, v_{i}, m_{i}\right)$ in $\varphi_{x}$. The weight of an edge from $v$ to $v^{\prime}$ in the graph of $\varphi_{x}^{d}$ is obtained by taking the sum of the products $m_{1} \cdots m_{d}$ over all paths of length $d$ from $v$ to $v^{\prime}$ in $\mathscr{G}_{x}$.

Figures A3 and A4 show the graphs of $\Phi_{x}^{2}$ and $\Phi_{x}^{3}$, respectively, and we see that for $\operatorname{deg} y=2$, we have $\Phi_{x}^{2} \equiv \Phi_{y}+2 q \cdot 1(\bmod \mathscr{}(K))$ and for $\operatorname{deg} y=3$, we have 

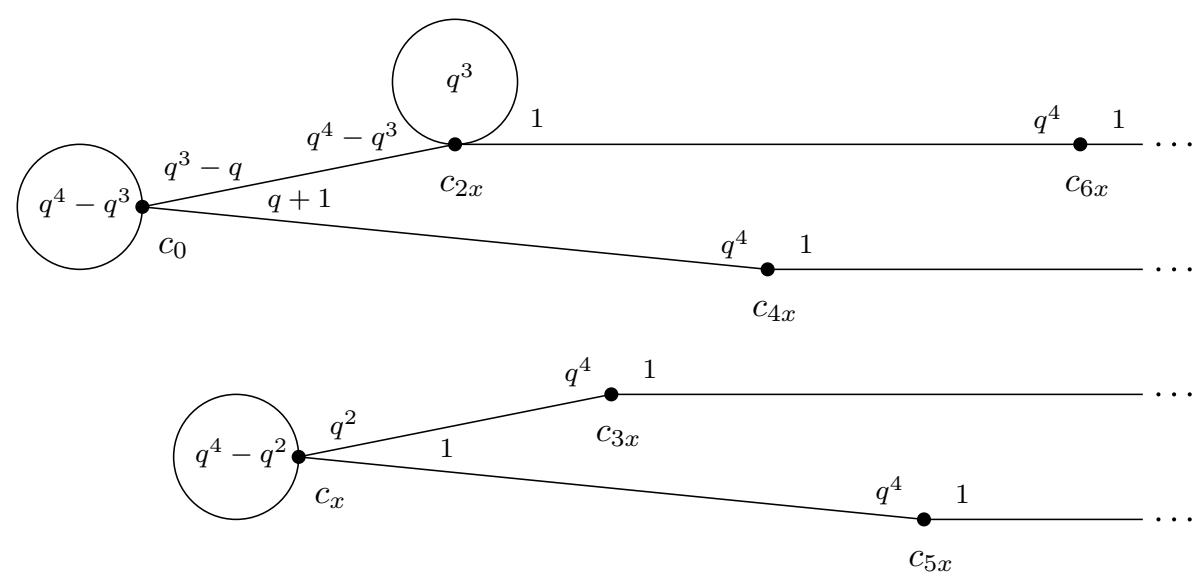

Figure A5. The graph of $\Phi_{y}$ for a place $y$ of degree 4 .

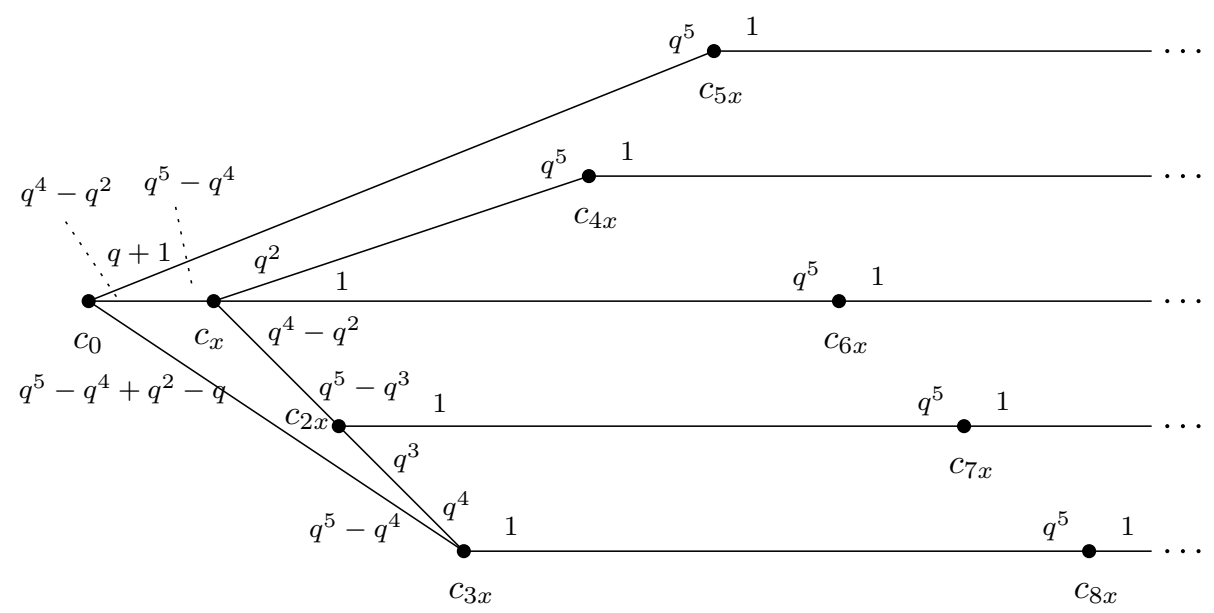

Figure A6. The graph of $\Phi_{y}$ for a place $y$ of degree 5 .

$\Phi_{x}^{3} \equiv \Phi_{y}+3 q \cdot \Phi_{x}(\bmod \mathscr{L}(K))$, where $\mathscr{g}(K)$ is the ideal of $\mathscr{H}_{K}$ of Hecke operators that operate trivially on $C^{0}\left(G_{F} \backslash G_{\AA}\right)$.

Example A.7 (the graphs of two ramified Hecke operators). It is also possible to determine examples for Hecke operators in $\mathscr{H}_{K^{\prime}}$ by elementary matrix manipulations, when $K^{\prime}<K$ is a subgroup of finite index. We will show two examples, which are illustrated in Figures A7 and A8. We omit the calculation, but only point out why the crucial differences between the two graphs occur. 


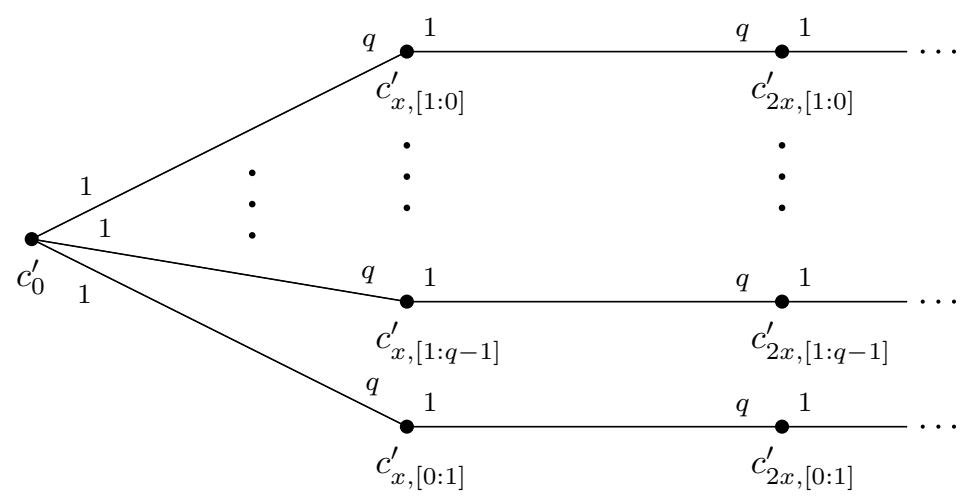

Figure A7. Graph of $\Phi_{y, e}^{\prime}$ as defined in Example A.7.

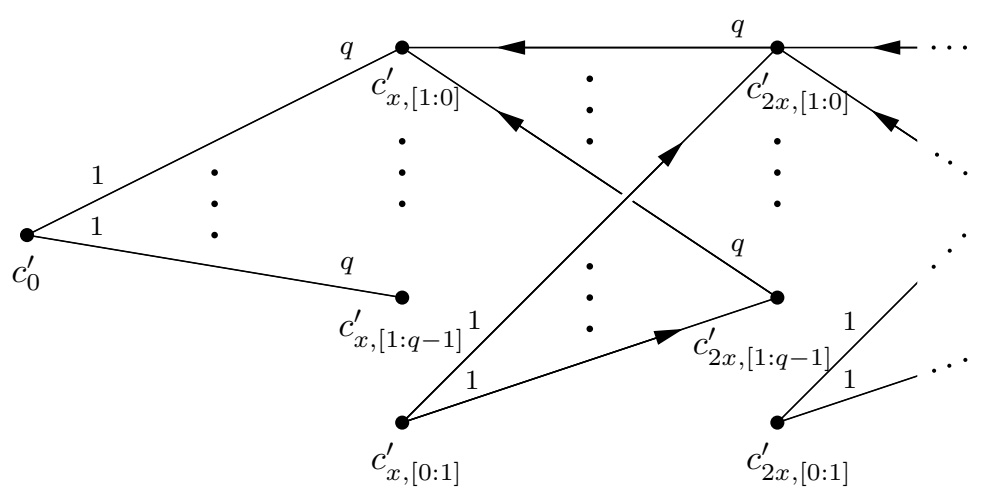

Figure A8. Graph of $\Phi_{x}^{\prime}$ as defined in Example A.7.

For $K^{\prime}=\left\{k \in K \mid k_{x} \equiv\left(\begin{array}{c}1 \\ { }^{1}\end{array}\right)\left(\bmod \pi_{x}\right)\right\}$, the fibers of the projection

$$
P: G_{F} \backslash G_{\AA} / K^{\prime} \rightarrow G_{F} \backslash G_{\AA} / K
$$

are given by $P^{-1}\left(c_{0}\right)=\left\{\left[p_{0}\right]\right\}$ and for positive $n$, by $P^{-1}\left(c_{n x}\right)=\left\{\left[p_{n x} \vartheta_{w}\right]\right\}_{w \in \mathbb{P}^{1}\left(\kappa_{x}\right)}$ with $\vartheta_{[1: c]}=\left(\begin{array}{cc}1 & c \\ 1\end{array}\right)$ and $\vartheta_{[0: 1]}=\left(\begin{array}{c}1 \\ 1\end{array}\right)$. The union of these fibers equals the set of vertices of a Hecke operator in $\mathscr{H}_{K^{\prime}}$. We shall denote the vertices by $c_{0}^{\prime}=\left[p_{0}\right]$ and $c_{n x, w}^{\prime}=\left[p_{n x} \vartheta_{w}\right]$ for $n \geq 1$ and $w \in \mathbb{P}^{1}\left(\kappa_{x}\right)$. Note that $G_{\mathbb{F}_{q}}=G_{\kappa_{x}}$ acts on $\mathbb{P}^{1}\left(\kappa_{x}\right)$ from the right, so if $\gamma \in G_{\mathbb{F}_{q}}$, then $w \mapsto w \gamma$ permutes the elements of $\mathbb{P}^{1}\left(\kappa_{x}\right)$.

The first Hecke operator $\Phi_{y, \gamma}^{\prime} \in \mathscr{H}_{K^{\prime}}$ that we consider is ( $\left.\operatorname{vol} K / \operatorname{vol} K^{\prime}\right)$ times the characteristic function of $K^{\prime}\left({ }^{\pi_{y}}{ }_{1}\right) \gamma K^{\prime}$, where $y$ is a degree one place different from $x$ and $\gamma \in G_{\mathbb{A}}$ is a matrix whose only nontrivial component is $\gamma_{x} \in G_{\mathbb{F}_{q}}$. (The factor ( $\operatorname{vol} K / \operatorname{vol} K^{\prime}$ ) is included to obtain integer weights.) Since $K^{\prime}\left({ }^{\pi_{y}}{ }_{1}\right) \gamma K^{\prime}$ is contained in $K\left(\begin{array}{ll}\pi_{y} & 1\end{array}\right) \gamma K$, the graph of $\Phi_{y, \gamma}^{\prime}$ relative to $K^{\prime}$ can have an edge from 
$v$ to $w$ only if $\mathscr{G}_{y}$ has an edge from $P(v)$ to $P(w)$. Because $K_{y}^{\prime}=K_{y}$, we argue as for $K$ that $K^{\prime}\left(\pi_{y}{ }_{1}\right) \gamma K^{\prime}=\bigsqcup_{w \in \mathbb{P}^{1}\left(\kappa_{y}\right)} \xi_{w} \gamma K^{\prime}$. Applying the same methods as in Example A.5, one obtains that

$$
\bigcup_{\Phi_{y, \gamma}^{\prime}, K^{\prime}}\left(c_{0}^{\prime}\right)=\left\{\left(c_{0}^{\prime}, c_{x, w}^{\prime}, 1\right)\right\}_{w \in \mathbb{P}^{1}\left(\kappa_{x}\right)}
$$

and for every $n \geq 1$ and $w \in \mathbb{P}^{1}\left(\kappa_{x}\right)$ that

$$
\mathcal{U}_{\Phi_{y, \gamma}^{\prime}, K^{\prime}}\left(c_{n x, w}^{\prime}\right)=\left\{\left(c_{n x, w}^{\prime}, c_{(n+1) x, w \gamma}^{\prime}, 1\right),\left(c_{n x, w}^{\prime}, c_{(n-1) x, w \gamma}^{\prime}, q\right)\right\} .
$$

For the case that $\gamma$ is equal to the identity matrix $e$, the graph is illustrated in Figure A7. Note that for general $\gamma$, an edge does not necessarily have an inverse edge since $w \gamma^{2}$ does not have to equal $w$.

The second Hecke operator $\Phi_{x}^{\prime} \in \mathscr{H}_{K^{\prime}}$ is $\left(\operatorname{vol} K / \operatorname{vol} K^{\prime}\right)$ times the characteristic function of $K^{\prime}\left(\begin{array}{ll}\pi_{x} & 1\end{array}\right) K^{\prime}$. This case behaves differently, since $K_{x}^{\prime}$ and $K_{x}$ are not equal; in particular, we have $K^{\prime}\left(\begin{array}{cc}\pi_{x} & 1\end{array}\right) K^{\prime}=\bigsqcup_{b \in \kappa_{x}}\left(\begin{array}{c}\pi_{x} b \pi_{x} \\ 1\end{array}\right) K^{\prime}$. This allows us to compute the edges as illustrated in Figure A8. Note that for $n \geq 1$, the vertices of the form $c_{n x,[1: 0]}^{\prime}$ and $c_{n x,[0: 1]}^{\prime}$ behave particularly.

\section{Acknowledgements}

This paper is extracted from my thesis [Lorscheid 2008]. First of all, I would like to thank Gunther Cornelissen for his advice during my graduate studies. I would like to thank Frits Beukers and Roelof Bruggeman for their numerous comments on a lecture series about my studies. I would like to thank the referee for his or her rich and detailed comments on the paper.

\section{References}

[Arason et al. 1992] J. K. Arason, R. Elman, and B. Jacob, "On indecomposable vector bundles", Comm. Algebra 20:5 (1992), 1323-1351. MR 93e:14051 Zbl 0769.14004

[Atiyah 1956] M. Atiyah, "On the Krull-Schmidt theorem with application to sheaves", Bull. Soc. Math. France 84 (1956), 307-317. MR 19,172b Zbl 0072.18101

[Bourbaki 1965] N. Bourbaki, Éléments de mathématique, Algèbre commutative, Chapitre 7: Diviseurs, Actualités Scientifiques et Industrielles 1314, Hermann, Paris, 1965. MR 41 \#5339 Zbl 0141.03501

[Frenkel 2004] E. Frenkel, "Recent advances in the Langlands program", Bull. Amer. Math. Soc. (N.S.) 41:2 (2004), 151-184. MR 2005e:11147 Zbl 1070.11051

[Gaitsgory 2003] D. Gaitsgory, "Informal introduction to geometric Langlands", pp. 269-281 in An introduction to the Langlands program (Jerusalem, 2001), edited by J. Bernstein and S. Gelbart, Birkhäuser, Boston, MA, 2003. MR 1990383 Zbl 1111.11308

[Gekeler 1995] E.-U. Gekeler, "Improper Eisenstein series on Bruhat-Tits trees", Manuscripta Math. 86:3 (1995), 367-391. MR 95m:11043 Zbl 0884.11025

[Gekeler 1997] E.-U. Gekeler, “On the Drinfeld discriminant function”, Compositio Math. 106:2 (1997), 181-202. MR 98e:11071 Zbl 0930.11031 
[Gekeler and Nonnengardt 1995] E.-U. Gekeler and U. Nonnengardt, "Fundamental domains of some arithmetic groups over function fields", Internat. J. Math. 6:5 (1995), 689-708. MR 96i:11043 Zbl 0858.11025

[Gelbart 1975] S. S. Gelbart, Automorphic forms on adèle groups, Annals of Mathematics Studies 83, Princeton University Press, 1975. MR 52 \#280 Zbl 0329.10018

[Harder and Narasimhan 1974/75] G. Harder and M. S. Narasimhan, "On the cohomology groups of moduli spaces of vector bundles on curves", Math. Ann. 212 (1974/75), 215-248. MR 51 \#509 Zbl 0324.14006

[Hartshorne 1977] R. Hartshorne, Algebraic geometry, Graduate Texts in Mathematics 52, Springer, New York, 1977. MR 57 \#3116 Zbl 0367.14001

[Kneser 1966] M. Kneser, "Strong approximation”, pp. 187-196 in Algebraic Groups and Discontinuous Subgroups (Boulder, CO, 1965), edited by A. Borel and G. D. Mostow, Amer. Math. Soc., Providence, R.I., 1966. MR 35 \#4225 Zbl 0201.37904

[Lang 1956] S. Lang, "Algebraic groups over finite fields", Amer. J. Math. 78 (1956), 555-563. MR 19,174a Zbl 0073.37901

[Laumon 1997] G. Laumon, Cohomology of Drinfeld modular varieties, II: Automorphic forms, trace formulas and Langlands correspondence, Cambridge Studies in Advanced Mathematics 56, Cambridge University Press, 1997. MR 98c:11045b Zbl 0870.14016

[Lorscheid 2008] O. Lorscheid, Toroidal automorphic forms for function fields, $\mathrm{PhD}$ thesis, University of Utrecht, 2008, available at http://igitur-archive.library.uu.nl.

[Lorscheid 2010] O. Lorscheid, "Toroidal automorphic forms for function fields", preprint, 2010. To appear in Israel J. Math. arXiv 1012.3223

[Lorscheid 2012] O. Lorscheid, "Automorphic forms for elliptic function fields", Math. Z. 272:3-4 (2012), 885-911. MR 2995144

[Margulis 1977] G. A. Margulis, "Cobounded subgroups in algebraic groups over local fields", Funkcional. Anal. i Priložen. 11:2 (1977), 45-57, 95. In Russian; translated in Functional Anal. Appl., 11:2 (1977), 119-122. MR 56 \#495

[Moore 1968] C. C. Moore, "Group extensions of p-adic and adelic linear groups", Inst. Hautes Études Sci. Publ. Math. 35 (1968), 157-222. MR 39 \#5575 Zbl 0159.03203

[Moreno 1985] C. J. Moreno, "Analytic proof of the strong multiplicity one theorem”, Amer. J. Math. 107:1 (1985), 163-206. MR 86m:22027 Zbl 0564.10035

[Prasad 1977] G. Prasad, "Strong approximation for semi-simple groups over function fields", Ann. of Math. (2) 105:3 (1977), 553-572. MR 56 \#2921 Zbl 0348.22006

[van der Put and Reversat 1997] M. van der Put and M. Reversat, "Automorphic forms and Drinfeld's reciprocity law", pp. 188-223 in Drinfeld modules, modular schemes and applications (AldenBiesen, 1996), edited by E.-U. Gekeler et al., World Sci. Publ., River Edge, NJ, 1997. MR 99j:11059 Zbl 0924.11051

[Schleich 1974] T. Schleich, Einige Bemerkungen zur Spektralzerlegung der Hecke-Algebra für die $\mathrm{PGL}_{2}$ über Funktionenkörpern, Bonner Mathematische Schriften 71, Mathematisches Institut, Universität Bonn, Bonn, 1974. MR 56 \#8759 Zbl 0345.10013

[Serre 2003] J.-P. Serre, Trees, Springer, Berlin, 2003. MR 2003m:20032 Zbl 1013.20001

Communicated by Edward Frenkel

Received 2011-04-11 Revised 2012-01-25 
lorschei@impa.br

Instituto Nacional de Matemática Pura e Aplicada, IMPA, Estrada Dona Castorina 110, 22460-320 Rio de Janeiro, RJ, Brazil

http://w3.impa.br/ lorschei/ 


\section{Algebra \& Number Theory}

msp.org/ant

\section{EDITORS}

MANAGING EDITOR

Bjorn Poonen

Massachusetts Institute of Technology

Cambridge, USA

\author{
EDITORIAL BOARD CHAIR \\ David Eisenbud \\ University of California \\ Berkeley, USA
}

\section{BOARD OF EDITORS}

Georgia Benkart

Dave Benson

Richard E. Borcherds

John H. Coates

J-L. Colliot-Thélène

Brian D. Conrad

Hélène Esnault

Hubert Flenner

Edward Frenkel

Andrew Granville

Joseph Gubeladze

Ehud Hrushovski

Craig Huneke

Mikhail Kapranov

Yujiro Kawamata

János Kollár

Yuri Manin

Barry Mazur

Philippe Michel
University of Wisconsin, Madison, USA

University of Aberdeen, Scotland

University of California, Berkeley, USA

University of Cambridge, UK

CNRS, Université Paris-Sud, France

University of Michigan, USA

Freie Universität Berlin, Germany

Ruhr-Universität, Germany

University of California, Berkeley, USA

Université de Montréal, Canada

San Francisco State University, USA

Hebrew University, Israel

University of Virginia, USA

Yale University, USA

University of Tokyo, Japan

Princeton University, USA

Northwestern University, USA

Harvard University, USA

École Polytechnique Fédérale de Lausanne
Susan Montgomery

Shigefumi Mori

Raman Parimala

Jonathan Pila

Victor Reiner

Karl Rubin

Peter Sarnak

Joseph H. Silverman

Michael Singer

Vasudevan Srinivas

J. Toby Stafford

Bernd Sturmfels

Richard Taylor

Ravi Vakil

Michel van den Bergh

Marie-France Vignéras

Kei-Ichi Watanabe

Efim Zelmanov
University of Southern California, USA

RIMS, Kyoto University, Japan

Emory University, USA

University of Oxford, UK

University of Minnesota, USA

University of California, Irvine, USA

Princeton University, USA

Brown University, USA

North Carolina State University, USA

Tata Inst. of Fund. Research, India

University of Michigan, USA

University of California, Berkeley, USA

Harvard University, USA

Stanford University, USA

Hasselt University, Belgium

Université Paris VII, France

Nihon University, Japan

University of California, San Diego, USA

\section{PRODUCTION}

production@msp.org

Silvio Levy, Scientific Editor

See inside back cover or msp.org/ant for submission instructions.

The subscription price for 2013 is US \$200/year for the electronic version, and \$350/year ( $\$ 40$, if shipping outside the US) for print and electronic. Subscriptions, requests for back issues and changes of subscribers address should be sent to MSP.

Algebra \& Number Theory (ISSN 1944-7833 electronic, 1937-0652 printed) at Mathematical Sciences Publishers, 798 Evans Hall \#3840, c/o University of California, Berkeley, CA 94720-3840 is published continuously online. Periodical rate postage paid at Berkeley, CA 94704, and additional mailing offices.

ANT peer review and production are managed by EditFLOW ${ }^{\circledR}$ from Mathematical Sciences Publishers.

\section{PUBLISHED BY}

mathematical sciences publishers

nonprofit scientific publishing

http://msp.org/

(C) 2013 Mathematical Sciences Publishers 


\section{Algebra \& Number Theory}

\section{Volume $7 \quad$ No. $1 \quad 2013$}

Powers of ideals and the cohomology of stalks and fibers of morphisms

MARC CHARDIN

Graphs of Hecke operators

OLIVER LORSCHEID

Group actions of prime order on local normal rings

FrANZ KIRÀLY and WERNER LÜTKEBOHMERT

On the arithmetic and geometry of binary Hamiltonian forms

JOUNI PARKKONEN and FRÉDÉRIC PAULIN

$L$-functions and periods of adjoint motives

MICHAEL HARRIS

Galois module structure of local unit groups

ROMYAR SHARIFI

On the invariant theory for tame tilted algebras

CALIN CHINDRIS

Period functions and cotangent sums

SANDRO BETTIN and BRIAN CONREY 